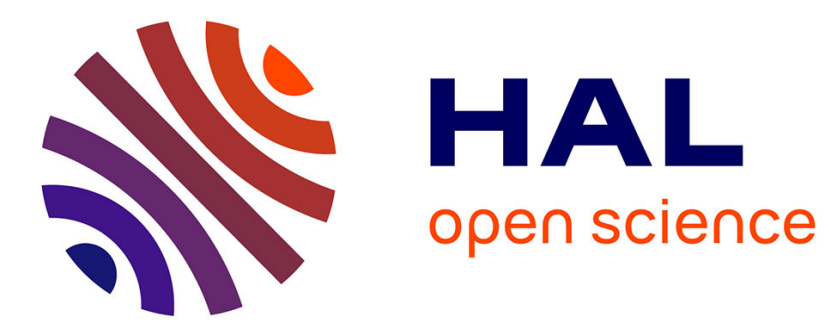

\title{
Lyapunov stability and performance analysis of the implicit discrete sliding mode control
}

\author{
Olivier Huber, Vincent Acary, Bernard Brogliato
}

\section{To cite this version:}

Olivier Huber, Vincent Acary, Bernard Brogliato. Lyapunov stability and performance analysis of the implicit discrete sliding mode control. IEEE Transactions on Automatic Control, 2016, 61 (10), pp.3016-3030. 10.1109/TAC.2015.2506991 . hal-01236159

\section{HAL Id: hal-01236159 \\ https://inria.hal.science/hal-01236159}

Submitted on 1 Feb 2016

HAL is a multi-disciplinary open access archive for the deposit and dissemination of scientific research documents, whether they are published or not. The documents may come from teaching and research institutions in France or abroad, or from public or private research centers.
L'archive ouverte pluridisciplinaire HAL, est destinée au dépôt et à la diffusion de documents scientifiques de niveau recherche, publiés ou non, émanant des établissements d'enseignement et de recherche français ou étrangers, des laboratoires publics ou privés. 


\title{
Lyapunov stability and performance analysis of the implicit discrete sliding mode control
}

\author{
Olivier Huber, Vincent Acary, Bernard Brogliato
}

\begin{abstract}
Discrete-time sliding mode controllers with an implicit discretization of the signum function are considered. With a proper choice of the equivalent part of the control, the resulting controller is shown to be Lyapunov stable with finitetime convergence of the sliding variable to 0 . The convergence of the control input, as the sampling period goes to 0 , to the continuous-time one is shown. The robustness with respect to matching perturbations is also investigated. The discretization performance in terms of the error order is studied for different discretizations of the equivalent part of the input. Numerical and experimental results illustrate and support the analysis.
\end{abstract}

Index Terms-sliding mode control, sampled-data system, discrete-time Lyapunov stability, implicit discretization, finitetime stability

\section{INTRODUCTION}

Discrete-time sliding mode control has been the object of many articles since several decades. The challenge of the chattering phenomenon, seen in simulations and experiments has sparked an intense activity in the past 30 years [1]-[8]. This concerns in particular the classical Equivalent-Control-Based Sliding-Mode Control (ECB-SMC), which consists of two subcontrollers: the state-continuous equivalent control $u^{e q}$ and the state-discontinuous control $u^{s}$. In these past research efforts, most of the focus was on the discontinuous part of the control, since it introduces numerical chattering. Several solutions to alleviate numerical chattering (that is solely due to the time discretization [9]-[13]) have been proposed [1]-[7], [14]-[16], most of them using a so-called quasi-sliding surface [5] or a boundary layer, associated with an explicit discretization of $u^{s}$. The works in [2] and [6] depart from these discretetime controllers and propose an algorithm which allows the sliding variable to take exactly the zero value at sampling times. They are however limited to scalar systems and require some stringent assumptions. Recently a new approach, introduced in [12] and [13], consists in implementing the discrete-time discontinuous input $u^{s}$ in an implicit form, while keeping its causality (i.e. the controller is nonanticipative). This input is at each sampling time the solution to a generalized, set-valued equation. In the simplest cases, the computation is reduced to a simple projection onto an interval. The core distinction between almost all previous discrete-time SMC controller and the approach in [12], [13] lies in the discretization method used for the argument of the signum function, respectively the explicit and implicit methods. Let us illustrate the difference

The authors acknowledge the support of the ANR grant CHASLIM (ANR11-BS03-0007).

The authors are with INRIA Grenoble Rhône-Alpes, BIPOP project-team, 655 avenue de l'Europe, Inovallée, 38334 Saint-Ismier, France. between the two discretizations with an academic example: $\dot{x}(t) \in-\alpha \operatorname{sgn}(x(t)), \alpha>0$. We use the differential inclusion framework as we let $\operatorname{sgn}(0)$ take any value in $[-1,1]$ (this is formally stated in Definition 1 as Sgn). An explicit discretization yields $x\left(t_{k+1}\right) \in x\left(t_{k}\right)-h \alpha \operatorname{sgn}\left(x\left(t_{k}\right)\right)$ whereas the implicit one yields $x\left(t_{k+1}\right) \in x\left(t_{k}\right)-h \alpha \operatorname{sgn}\left(x\left(t_{k+1}\right)\right)$. As long as $\left|x\left(t_{k}\right)\right| \gg h \alpha$, there is no difference between the two methods. But if $\left|x\left(t_{k}\right)\right|<\alpha h$, then the behavior changes with the type of discretization. With $0<x\left(t_{k}\right)<h \alpha$, in the explicit case, $x\left(t_{k+1}\right) \in x\left(t_{k}\right)-h \alpha \operatorname{sgn}\left(x\left(t_{k}\right)\right)<0$. The sign of the state changes at every $t_{k}$, leading to the wellknown chattering phenomenon. Meanwhile in the implicit case, the control algorithm guarantees $x\left(t_{k+1}\right)=0$ by choosing $\operatorname{sgn}\left(x\left(t_{k+1}\right)\right)=x\left(t_{k}\right) /(\alpha h)<1$. The implicit discretization of the signum function is rigorously presented in Section IV.

In this paper the following topics are tackled:

- the stability of the sliding variable using Lyapunov functions within the Variational Inequalities (VI) framework, and its finite-time convergence, with an estimation of the number of steps to reach 0 .

- the convergence of the discrete-time control input as the sampling time goes to 0: with an implicit discretization, the discrete-time control input converges to the selection of the continuous-time one.

- the robustness and the insensitivity w.r.t an increase of the gain of the implicit discrete-time controller during the sliding phase.

- the comparison of different discretization methods for the equivalent part of the control using simulation results to underline the influence of those methods on the closedloop behavior.

- the experimental validation of several theoretical results.

To the best of our knowledge, these issues have not been addressed before, and they significantly extend the results in [12] and [13]. Furthermore the conditions for the stability and robustness properties are shown to be close to the continuoustime ones. Note that the knowledge of the system parameters is required, and the relaxation of this assumption (which produces an equivalent disturbance that is not globally bounded) is left as a future work.

In the next section, we introduce the class of systems and the notations. In Section III we briefly recall the ECB-SMC theory. Then we propose a new discrete-time SMC scheme in Section IV. Stability and convergence results are derived in Section V. Section VI presents some experimental results on an inverted pendulum on a cart, which highlight the benefit of the implicit discretization with respect to the explicit one. 
Simulation results using different time-discretization methods are shown in Section VII, to illustrate the possible different behaviors of the closed-loop system. Section VIII is dedicated to the discrete error analysis of various controllers of Section IV and VII. Conclusions end the paper in Section IX, and some proofs are given in the Appendices.

\section{PRELIMINARIES}

In the sequel, we consider well-posed systems (in the sense of Filippov [17]) of the form

$$
\left\{\begin{array}{l}
\dot{x}(t)=A x(t)+B u(t)+B \xi(t, x) \\
u(t)=u^{e q}(t)+u^{s}(t) \\
\sigma(t):=C x(t) \\
-u^{s}(t) \in \alpha \operatorname{Sgn}(\sigma(x(t))),
\end{array}\right.
$$

with $x(t) \in \mathbb{R}^{n}, u(t) \in \mathbb{R}^{p}, \sigma(t) \in \mathbb{R}^{p}, C \in \mathbb{R}^{p \times n}$, and $\alpha>0$. The function $\sigma$ is called the sliding variable, the matched and uniformly bounded disturbance is denoted as $\xi$, and Sgn is formally introduced in Definition 1. The perturbation $\xi$ is supposed to be at least continuous: noisy processes are not considered in this paper. When $\xi \equiv 0$, the system is said to be nominal. The method used to discretize the dynamics is called Zero-Order Hold $(\mathrm{ZOH})$, also known as exact sampled-data representation, see [18, Chapter $2 \mathrm{~d}]$.

Notations: Let $\mathbf{x}: \mathbb{R}_{+} \times \mathbb{R}^{p} \times \mathbb{R}^{n} \rightarrow \mathbb{R}^{n}$ be the solution of system (1), $x:=\mathbf{x}\left(\cdot, u, x_{0}\right)$ is the solution associated with a continuous-time control $u$ and an initial state $x_{0} \in \mathbb{R}^{n}$, while $\bar{x}:=\mathbf{x}\left(\cdot, \bar{u}, x_{0}\right)$ is the solution with a step function $\bar{u}$ and the same initial state. In the latter case, we denote by $\bar{\sigma}:=C \bar{x}$ the sliding variable. The control values change at predefined time instants $t_{k}$, defined for all $k \in \mathbb{N}: t_{k}:=$ $t_{0}+k h, t_{0}, h \in \mathbb{R}_{+}$. The scalar $h$ is called the sampling period. We denote $\bar{x}_{k}:=\bar{x}\left(t_{k}\right)$ and $\bar{\sigma}_{k}:=\bar{\sigma}\left(t_{k}\right)$ for all $k \in$ $\mathbb{N}$. For all $y \in \mathbb{R}^{r},\|y\|_{\infty}=\max _{i}\left|y_{i}\right|$. For all $M \in \mathbb{R}^{r \times s}$, $\|M\|_{\infty}=\max _{i} \sum_{j}\left|M_{i j}\right|$. Let $w: \mathbb{R} \rightarrow \mathbb{R}^{r}$ and $S$ be any interval in $\mathbb{R},\|w\|_{\infty, S}=\max _{i} \operatorname{ess}_{\sup _{t \in S}}\left|w_{i}(t)\right|$. Let $\langle\cdot, \cdot\rangle$ denote the standard inner product in a Euclidean space and $\|\cdot\|$ the vector norm based upon it. The matrix norm $\|\cdot\|$ is the spectral norm (i.e. the largest singular value). Let sgn be the classical single-valued signum function: for all $x>$ $0, \operatorname{sgn}(x)=1, \operatorname{sgn}(-x)=-1$ and $\operatorname{sgn}(0)=0$.

Definition 1 (Multivalued signum function). Let $x \in \mathbb{R}$. The multivalued signum function $\mathrm{Sgn}: \mathbb{R} \rightrightarrows \mathbb{R}$ is defined as:

$$
\operatorname{Sgn}(x)= \begin{cases}\{1\} & x>0 \\ \{-1\} & x<0 \\ {[-1,1]} & x=0\end{cases}
$$

If $x \in \mathbb{R}^{n}$, then the multivalued signum function Sgn: $\mathbb{R}^{n} \rightrightarrows$ $\mathbb{R}^{n}$ is defined as: for all $j=1, \ldots, n,(\operatorname{Sgn}(x))_{j}:=\operatorname{Sgn}\left(x_{j}\right)$.

Definition 2. Let $f: \mathbb{R}^{n} \times \mathbb{R} \rightarrow \mathbb{R}^{p}$ and $l \in \mathbb{R}$. One has $f=\mathcal{O}\left(h^{l}\right)$ if for all $x \in \mathbb{R}^{n}$, there exists $c \in \mathbb{R}^{p}$ such that $f(x, h) / h^{l} \rightarrow c$ as $h \rightarrow 0$.

We make use of results stemming from the study of complementarity problems. Let us introduce some related concepts now.
Definition 3. [19, p. 147] Let $M \in \mathbb{R}^{n \times n}$. $M$ is a $\mathbf{P}$-matrix if for all $x \in \mathbb{R}^{n}$ such that for all $i \in\{1, \ldots, n\}, x_{i}(M x)_{i} \leq 0$, then $x=0$.

Lemma 1. [19, p. 147] Let $M \in \mathbb{R}^{n \times n}$. If $M$ is positive definite, then $M$ is a $\mathbf{P}$-matrix.

\section{ThE EQUIVALENT-BASED CONTINUOUS-TIME SLIDING-MODE CONTROLLER}

Let us assume that $C$ is chosen such that the decoupling matrix $C B$ is full rank. The dynamics of the sliding variable in the nominal system (1) (that is with $\xi(t)=0$ ) is

$$
\dot{\sigma}(t)=C A x(t)+C B u^{e q}(t)+C B u^{s}(t) .
$$

The control law $u^{e q}$ is designed such that the system stays on the sliding surface once it has been reached (in other word $u^{e q}$ renders the sliding surface invariant with $\left.u^{s} \equiv 0\right)$ :

$$
\dot{\sigma}(t)=0 \text { and } u^{s}(t)=0 \Rightarrow u^{e q}(t)=-(C B)^{-1} C A x(t) .
$$

With this equivalent controller, the sliding variable dynamics reduces to

$$
\left\{\begin{aligned}
\dot{\sigma}(t) & =C B u^{s}(t) \\
-u^{s}(t) & \in \alpha \operatorname{Sgn}(\sigma(t)) .
\end{aligned}\right.
$$

The dynamics in (1) can be rewritten as

$$
\begin{array}{r}
\dot{x}(t)=\left(I-B(C B)^{-1} C\right) A x(t)+B u^{s}(t) \\
\Longleftrightarrow \dot{x}(t)=\Pi A x(t)+B u^{s}(t),
\end{array}
$$

with $\Pi:=I-B(C B)^{-1} C$. Two interesting properties of $\Pi$ are $C \Pi=0$ and $\Pi$ is a projector [20]. Taking the integral form of system (5) yields the relation

$$
x(t)=\Phi\left(t, t_{0}\right) x\left(t_{0}\right)+\int_{t_{0}}^{t} \Phi(t, \tau) B u^{s}(\tau) \mathrm{d} \tau,
$$

with $\Phi\left(t, t_{0}\right)=e^{\Pi A\left(t-t_{0}\right)}$ the state transition matrix for the system (5). Some properties of $\Phi$ are given in the following lemma.

Lemma 2. One has $\dot{\Phi}\left(t, t_{0}\right)=\Pi A \Phi\left(t, t_{0}\right), \Phi\left(t_{0}, t_{0}\right)=I$, and $C \Phi\left(t, t_{0}\right)=C$ for all $t \geq t_{0}$.

Proof. It is true that $C \dot{\Phi}\left(t, t_{0}\right)=0$ so $C \Phi\left(t, t_{0}\right)=$ $C \Phi\left(t_{0}, t_{0}\right)=C$ for all $t \geq t_{0}$.

\section{DISCRETE-TIME CONTROLLERS}

\section{A. Discretization methods to obtain discrete-time controllers}

From now on, $\bar{u}^{e q}$ and $\bar{u}^{s}$ are sampled control laws defined as right-continuous step functions:

$$
\bar{u}^{e q}(t)=\bar{u}_{k}^{e q} \quad \text { and } \quad \bar{u}^{s}(t)=\bar{u}_{k}^{s} \quad \text { for } t \in\left[t_{k}, t_{k+1}\right) .
$$

The goal of the discretization process is to choose the sequences $\left\{\bar{u}_{k}^{e q}\right\}$ and $\left\{\bar{u}_{k}^{s}\right\}$ such that the discrete-time system exhibits properties as close as possible to the ones with a continuoustime set-valued controller.

Integrating the nominal version of (1) over $\left[t_{k}, t_{k+1}\right)$ and using the expression in (6), one obtains the $\mathrm{ZOH}$ discretization of the system:

$$
\bar{x}_{k+1}=e^{A h} \bar{x}_{k}+B^{*} \bar{u}_{k}^{e q}+B^{*} \bar{u}_{k}^{s},
$$


with $\quad B^{*}:=\int_{t_{k}}^{t_{k+1}} e^{A\left(t_{k+1}-\tau\right)} B \mathrm{~d} \tau$.

Suppose that the implicit discretization shown in (23b) below, introduced in [12] and [13], is used to discretize the discontinuous part of the controller. Both the analysis and the computation of the control law involve an auxiliary square subsystem combining the dynamics of the sliding variable and the nonsmooth control law:

$$
\left\{\begin{array}{l}
\widetilde{\sigma}_{k+1}=\bar{\sigma}_{k}+C B^{*} \bar{u}_{k}^{s} \\
-\bar{u}_{k}^{s} \in \alpha \operatorname{Sgn}\left(\widetilde{\sigma}_{k+1}\right),
\end{array}\right.
$$

with two unknowns: $\widetilde{\sigma}_{k+1}$ and $\bar{u}_{k}^{s}$. This system can be seen as the discrete counterpart of (4). The use of $\widetilde{\sigma}_{k+1}$ instead of $\bar{\sigma}_{k+1}$ is deliberate in order to highlight that in general the two quantities are different even in the nominal case: the state dynamics is given by (7) with $\bar{u}_{k}^{e q}$ usually given by the discretization of its continuous-time version (3). Hence nothing guarantees that $C\left(e^{A h} \bar{x}_{k}+B^{*} \bar{u}_{k}^{e q}\right)=\bar{\sigma}_{k}$. We take care of this design issue in the last part of this section. But before let us show how we can transform (9) into a problem with only one unknown, which is better suited for the computation of the control law.

\section{B. Definition and properties of the implicitly discretized discontinuous control input}

We analyze the system (9) using the Affine Variational Inequalities (AVI) formalism [21], departing from the approach in [12] and [13] where the tools mostly came from Numerical Analysis. The closely related Linear Complementarity Problem (LCP) framework was previously also used, but only for the numerical computations. Let $\mathcal{N}_{[-\alpha, \alpha]^{p}}(\lambda)$ be the normal cone to the box $[-\alpha, \alpha]^{p}$ at $\lambda$, that is $\mathcal{N}_{[-\alpha, \alpha]^{p}}(\lambda)=\left\{d \in \mathbb{R}^{p} \mid\langle d, y-\right.$ $\left.\lambda\rangle \leq 0, \forall y \in[-\alpha, \alpha]^{p}\right\}$. The relation $-\bar{u}_{k}^{s} \in \operatorname{Sgn}\left(\widetilde{\sigma}_{k+1}\right) \Longleftrightarrow$ $-\widetilde{\sigma}_{k+1} \in \mathcal{N}_{[-\alpha, \alpha]^{p}}\left(\bar{u}_{k}^{s}\right)$ enables us to transform (9) into the generalized equation:

$$
0 \in \bar{\sigma}_{k}+C B^{*} \bar{u}_{k}^{s}+\mathcal{N}_{[-\alpha, \alpha]^{p}}\left(\bar{u}_{k}^{s}\right) .
$$

Using this transformation, only the unknown input remains. The inclusion (10) is satisfied if and only if $\bar{u}_{k}^{s}$ is the solution of the AVI: Find $z \in[-\alpha, \alpha]^{p}$ such that

$$
(y-z)^{T}\left(\bar{\sigma}_{k}+C B^{*} z\right) \geq 0, \quad \forall y \in[-\alpha, \alpha]^{p} .
$$

Let $\operatorname{SOL}\left(C B^{*}, C \bar{x}_{k}\right)$ denote the set of all solutions to the AVI (11).

\section{Lemma 3. The AVI (11) has always a solution.}

Proof. Since the mapping $z \mapsto C B^{*} z+\bar{\sigma}_{k}$ is continuous and the control set $[\alpha, \alpha]^{p}$ is compact, we can apply the Corollary 2.2.5, p. 148 in [21].

Lemma 4. The AVI (11) has a unique solution for all $\bar{\sigma}_{k} \in \mathbb{R}^{p}$ if and only if $C B^{*}$ is a $\mathbf{P}$-matrix.

Proof. Using Theorem 4.3.2 p. 372 and Example 4.2.9 p. 361 in [21] yields the result.

The matrix $C$ is a design variable, and it should be chosen such that $C B^{*}>0$ (and therefore is a $\mathbf{P}$-matrix). Lemma 6 below relates the positive-definiteness of $C B$ and $C B^{*}$. This AVI-based approach enables us to analyze a larger class of systems, compared to previous approaches where it is supposed that $C B^{*}$ is scalar as in [6]. The solution $\bar{u}_{k}^{s}$ is a function of $\bar{\sigma}_{k}$ (hence $\bar{x}_{k}$ ) and if $C B^{*}$ is a $\mathbf{P}$-matrix, the solution map $C \bar{x}_{k} \mapsto \bar{u}_{k}^{s}=\operatorname{SOL}\left(C B^{*}, \bar{\sigma}_{k}\right)$ is Lipschitz continuous. The computation of the control input value consists in solving (10): when the control is scalar or if $C B^{*}$ is diagonal, a solution can be computed as a simple orthogonal projection: with $\bar{v}_{k}=-\left(C B^{*}\right)^{-1} \bar{\sigma}_{k}$, we have $\bar{u}_{k}^{s}=\operatorname{proj}_{[-\alpha, \alpha]^{p}}\left(\bar{v}_{k}\right)$. This is equivalent to applying this rule component-wise: if $\left(\bar{v}_{k}\right)_{i}>\alpha,\left(\bar{u}_{k}^{s}\right)_{i}=\alpha$; else if $\left(\bar{v}_{k}\right)_{i}<-\alpha,\left(\bar{u}_{k}^{s}\right)_{i}=-\alpha$; else $\left(\bar{u}_{k}^{s}\right)_{i}=\left(\bar{v}_{k}\right)_{i}$. Otherwise a solution to (10) can be found using Lemke's algorithm or a reformulation as a quadratic problem may be used, see [13]. More details on the numerical aspects and solvers for this kind of problems can be found in [19], [22] and [23]. The AVI (10) highlights that the controller is non-anticipative (i.e. causal). Notice that the nominal model is used to compute the set-valued part of the controller, since both $A$ and $B$ are needed to calculate $B^{*}$ in (8). It is clear however on one hand that the ECB-SMC controller always requires the a priori knowledge of both $A$ and $B$ to calculate $u^{e q}$ in (3), whatever the used discretization (see (22a), (22b) and (22c) below). On the other hand this seems unavoidable if the $\mathrm{ZOH}$ discretization of the plant is used (see [13, Equation (16)] for the Euler discretization).

Remark 1. When $\bar{u}_{k}^{s}$ is in the interior of $[-\alpha, \alpha]^{p}$, we say that the closed-loop system is in the discrete-time sliding phase. The inclusion $-\widetilde{\sigma}_{k+1} \in \mathcal{N}_{[-\alpha, \alpha]^{p}}\left(\bar{u}_{k}^{s}\right)$ implies that in this case the normal cone is reduced to the singleton $\{0\}$. Thus the sliding variable $\widetilde{\sigma}$ is zero in the discrete-time sliding phase (however in general $\bar{\sigma}$ does not vanish).

Now that we have discussed the existence and uniqueness properties of solutions to (9) as well as methods to compute them, we need to tackle the computation of the equivalent part of the control.

\section{Exact discrete equivalent control}

Let us complete this sliding mode control scheme for a discrete-time LTI plant by providing the equivalent part of the control. As showed in (4), in continuous time, $u^{e q}$ is defined such that the dynamics of the sliding variable depends only on the input $u^{s}$. Mimicking the continuous-time design, we start from (7) and multiplying by $C$, we obtain in discrete-time the relation

$$
\bar{\sigma}_{k+1}=C \bar{x}_{k+1}=C e^{A h} \bar{x}_{k}+C B^{*} \bar{u}_{k}^{e q}+C B^{*} \bar{u}_{k}^{s} .
$$

Our design objective is to make $\bar{\sigma}_{k+1}$ depend only on $\bar{\sigma}_{k}$ and $\bar{u}^{s}$. Hence we want that $C e^{A h} \bar{x}_{k}+C B^{*} \bar{u}_{k}^{e q}=\bar{\sigma}_{k}$, which gives

$$
\bar{u}_{k}^{e q}=\left(C B^{*}\right)^{-1} C\left(I-e^{A h}\right) \bar{x}_{k} .
$$

If we substitute this expression for $\bar{u}_{k}^{e q}$ in (12), then, as expected, we obtain $\bar{\sigma}_{k+1}=\bar{\sigma}_{k}+C B^{*} \bar{u}_{k}^{s}$. Using the implicitly discretized discontinuous part of the control $u_{k}^{s} \in \alpha \operatorname{Sgn}\left(\bar{\sigma}_{k+1}\right)$, the discrete-time sliding variable dynamics is

$$
\left\{\begin{array}{l}
\bar{\sigma}_{k+1}=\bar{\sigma}_{k}+C B^{*} \bar{u}_{k}^{s} \\
-\bar{u}_{k}^{s} \in \alpha \operatorname{Sgn}\left(\bar{\sigma}_{k+1}\right) .
\end{array}\right.
$$


This system has the same structure as in (9), although with the important difference that $\widetilde{\sigma}_{k+1}=\bar{\sigma}_{k+1}$. This system is studied in the next section, where its finite-time stability and robustness is analysed. Let us first state the following result.

Lemma 5. If $C B^{*}$ is a $\mathbf{P}$-matrix, then the only equilibrium pair of the system $(14)$ is $\left(\bar{\sigma}_{*}, \bar{u}_{*}^{s}\right)=(0,0)$.

Proof. A pair $\left(\bar{\sigma}, \bar{u}^{s}\right)$ is an equilibrium of (14) if and only if $C B^{*} \bar{u}^{s}=0$. If $C B^{*}$ is a $\mathbf{P}$-matrix, then it has full-rank and $C B^{*} \bar{u}^{s}=0$ is equivalent to $\bar{u}^{s}=0$. From the definition of the Sgn multifunction in (2), this is only possible if $\bar{\sigma}=0$.

To sum up, with the proposed scheme the two control inputs are

$$
\left\{\begin{array}{l}
\bar{u}_{k}^{e q}=\left(C B^{*}\right)^{-1} C\left(I-e^{A h}\right) \bar{x}_{k} \\
\bar{u}_{k}^{s} \quad \text { solution of (14). }
\end{array}\right.
$$

The complete controller is nonanticipative since $\bar{u}_{k}^{e q}$ depends only on the model parameters and $\bar{x}_{k}$. Moreover $\bar{u}_{k}^{s}$ is the unique solution to (14) given that $C B^{*}>0$, using similar arguments as in Lemma 4. This controller retains the structure of the continuous-time sliding mode controller. It is different from the approach that can be found in [4] or [24] since in our case, the equivalent part $u^{e q}$ is not chosen as the solution to a deadbeat control problem. As a result, the magnitude of the control input in (14) is $\mathcal{O}(1)$ with respect to the sampling period $h$, whereas it is $\mathcal{O}\left(h^{-1}\right)$ in the deadbeat case, see [24]. In [3], this expression for the equivalent control was already derived, when the sliding variable is scalar.

\section{STABILITY AND CONVERGENCE PROPERTIES}

In this section, we investigate the stability of the auxiliary system (14) and some convergence properties of the control input $\bar{u}^{s}$ when the sampling period tends to 0 . In the nominal case we are able to prove convergence of the sliding variable to 0 , using Lyapunov technique, under some structural conditions that match closely the ones for the continuous-time sliding mode controller. In the case where the dynamics include matched perturbations, we show how the proposed controller attenuates their effects and that if the controller action is large enough, the system remains in a neighborhood of the sliding manifold. We study the convergence of the control input since for control theorist it is a crucial variable and it received little attention in discretization studies of differential inclusions. Those results also underline the difference between the implicit and explicit discretizations. All the results we get on the system (14) are valid for the closed-loop system (7) and (15), thanks to the structure of the controller introduced in the previous section.

\section{A. Stability in the nominal case}

In this subsection, the stability of the system (14) is analyzed. Note that the mapping $\operatorname{Sgn}(\cdot)$, as introduced in Definition 1, has the following properties:

$$
\begin{aligned}
&\left\langle v_{1}-v_{2}, x_{1}-x_{2}\right\rangle \geq 0, \forall v_{i} \in \operatorname{Sgn}\left(x_{i}\right), i=1,2 \\
& 0 \in \operatorname{Sgn}(x) \Longleftrightarrow \quad x=0 .
\end{aligned}
$$

Property (16) is known as the monotonicity of the Sgn setvalued function, see [25, Chap. 12]. The positive-definitiveness property of $C B^{*}$ is pivotal to the results presented in this section. Even if it is not explicit with the current notations, $C B^{*}$ depends on the sampling period $h$. The following lemma gives some insight of when this condition is fulfilled.

Lemma 6. Suppose that $C B$ is positive definite. There exists an interval $\mathcal{I}=\left(0, h^{*}\right] \subset \mathbb{R}_{+}, h^{*}>0$, such that if the sampling period $h \in \mathcal{I}$, then $C B^{*} / h$ and $C B^{*}$ are positive definite.

Proof. Let $h>0, C B_{s}$ and $C B_{s}^{*}$ be the symmetric parts of $C B$ and $C B^{*}$, respectively. Let $\Delta:=C B_{s}^{*} / h-C B_{s}=$ $\sum_{l=1}^{\infty} \frac{C A^{l} B+B^{T}\left(A^{l}\right)^{T} C^{T}}{2(l+1) !} h^{l}=\mathcal{O}(h)$. Since $C B_{s}$ is symmetric, it is also normal. Hence we can apply Corollary 4.2.16, p. 405 in [26], which yields that for any eigenvalue $\mu$ of $C B_{s}^{*} / h$, $\min _{\lambda}|\lambda-\mu| \leq\|\Delta\|$, with $\lambda$ an eigenvalue of $C B_{s}$ and $\|\cdot\|$ the spectral norm. By definition, $\Delta$ is a symmetric matrix with real entries. Hence $\|\Delta\|=\delta_{\max }$, the largest module of any eigenvalue of $\Delta$. Let $\gamma>0$ be the smallest eigenvalue of $C B_{s}$. If $\delta_{\max }<\gamma$, then every eigenvalue of $C B_{s}^{*} / h$ is positive and since $C B_{s}^{*} / h$ is by definition symmetric, $C B_{s}^{*} / h$ is positive definite. It is easy to see that $\Delta \rightarrow 0$ as $h \rightarrow 0$ and that $\Delta$ depends continuously on $h$. Therefore by Corollary 4.2.4, p. 399 in [26], the eigenvalues of $\Delta$ are continuous functions of $h$. Then it is always possible to find $h^{*}$ such that $\delta_{\max }<\gamma$ for all $0<h<h^{*}$, which implies that $C B_{s}^{*} / h$ is positive definite as well as $C B_{s}^{*}$.

Let us investigate the Lyapunov stability of (14) with a candidate function inspired by the one presented in [27] in the continuous-time case. It is also possible to use a quadratic function, but this would require $C B^{*}$ to be symmetric.

Lemma 7. If $C B^{*}$ is positive definite, then the equilibrium state $\bar{\sigma}^{*}=0$ of (14) is globally Lyapunov stable.

Proof. Let $V\left(\bar{\sigma}_{k}\right):=\alpha\left\|\bar{\sigma}_{k}\right\|_{1}=-\left(\bar{u}_{k-1}^{s}\right)^{T} \bar{\sigma}_{k}$ be the candidate Lyapunov function, and $-\bar{u}_{k-1}^{s} \in \alpha \operatorname{Sgn}\left(\bar{\sigma}_{k}\right)$. The function $V$ is positive definite, radially unbounded, and decrescent. Let us study the evolution of $V$ :

$$
\begin{gathered}
V\left(\bar{\sigma}_{k+1}\right)-V\left(\bar{\sigma}_{k}\right)=-\left(\bar{u}_{k}^{s}\right)^{T} \bar{\sigma}_{k+1}+\left(\bar{u}_{k-1}^{s}\right)^{T} \bar{\sigma}_{k} \\
=-\left(\bar{u}_{k}^{s}\right)^{T}\left(\bar{\sigma}_{k}+C B^{*} \bar{u}_{k}^{s}\right)+\left(\bar{u}_{k-1}^{s}\right)^{T} \bar{\sigma}_{k} \\
=-\left(\bar{u}_{k}^{s}\right)^{T} C B^{*} \bar{u}_{k}^{s}+\left\langle\bar{u}_{k-1}^{s}-\bar{u}_{k}^{s}, \bar{\sigma}_{k}\right\rangle .
\end{gathered}
$$

The first term is always nonpositive with the hypothesis on $C B^{*}$. For the second term, let us recall that from the generalized equation (10), we have $-\bar{\sigma}_{k} \in \mathcal{N}_{[-\alpha, \alpha]^{p}}\left(\bar{u}_{k-1}\right)$. By definition this amounts to $\left\langle y-\bar{\sigma}_{k}\right\rangle \geq 0$ for all $y \in[-\alpha, \alpha]^{p}$, implying that the second term is always nonpositive.

The first term vanishes if and only if $\bar{u}_{k}^{s}=0$, which by (17) yields that $\bar{\sigma}_{k+1}=0$. Using (9) we have $\bar{\sigma}_{k}=0$, ending the proof.

Proposition 1. If the hypothesis of Lemma 7 is satisfied, then the fixed point $(\bar{\sigma}, \bar{u})=(0,0)$ of (14) is globally finite-time Lyapunov stable.

Proof. From the proof of Lemma 7, we know that $V\left(\bar{\sigma}_{k+1}\right)-$ $V\left(\bar{\sigma}_{k}\right)$ is equal to $-\left(\bar{u}_{k}^{s}\right)^{T} C B^{*} \bar{u}_{k}^{s}$ plus a nonpositive term. 
Since $C B^{*}$ is positive definite, it holds that $-\left(\bar{u}_{k}^{s}\right)^{T} C B^{*} \bar{u}_{k}^{s} \leq$ $-\beta\left\|\bar{u}_{k}^{s}\right\|^{2}$, with $\beta>0$ the smallest eigenvalue of $C B_{s}^{*}$. Note that if $\bar{\sigma}_{k+1} \neq 0$, then $\left\|\bar{u}_{k}^{s}\right\| \geq \alpha$ and $V\left(\bar{\sigma}_{k+1}\right)-V\left(\bar{\sigma}_{k}\right) \leq$ $-\alpha^{2} \beta$. Iterating, one obtains $V\left(\bar{\sigma}_{k+1}\right)-V\left(\bar{\sigma}_{0}\right) \leq-k \alpha^{2} \beta$. Let $k_{0}:=\left\lceil V\left(\bar{\sigma}_{0}\right) / \beta \alpha^{2}\right\rceil$ and suppose that $V\left(\bar{\sigma}_{k_{0}+1}\right) \neq 0$. Then $V\left(\bar{\sigma}_{k_{0}+1}\right)-V\left(\bar{\sigma}_{0}\right) \leq-k_{0} \alpha^{2} \beta \leq-V\left(\bar{\sigma}_{0}\right)$. This yields $V\left(\bar{\sigma}_{k_{0}+1}\right) \leq 0$, which implies $V\left(\bar{\sigma}_{k_{0}+1}\right)=0$. Hence $\bar{\sigma}_{k_{0}+1}=$ 0 and $\bar{\sigma}_{k}=0$ for all $k>k_{0}$.

To the best of our knowledge, these proofs of Lyapunov stability in the discrete-time case are new and have never been done before for discrete-time SMC.

Remark 2. Practical stabilization of delayed systems with a $\mathrm{ZOH}$ discretization and a sliding mode controller is analysed in [28]. Since the implicit method has some kind of predictive capabilities, it will be worth investigating the influence of an implicit discretization on this class of systems.

\section{B. Stability in the perturbed case}

Let us now consider the case when a matched perturbation $\xi$ is acting on the system, as in (1), which subsumes system uncertainties, unmodeled dynamics and external perturbations with this particular structure. It contributes to the $\mathrm{ZOH}$ discretized dynamics via an additive term

$$
p_{k}:=\int_{t_{k}}^{t_{k+1}} e^{A\left(t_{k+1}-\tau\right)} B \xi(\tau, x) \mathrm{d} \tau
$$

which is also added to the sliding variable dynamics $\bar{\sigma}_{k}$ in (12) (13):

$$
\bar{\sigma}_{k+1}=\bar{\sigma}_{k}+C B^{*} \bar{u}_{k}^{s}+C p_{k},
$$

with $\bar{u}_{k}^{s}$ the unique solution of the generalized equation (9). Although the system will never reach and stay on the sliding manifold as in the continuous-time case, we shall see that it enters the discrete-time sliding phase and stays in it, see Remark 1. Let us first present a technical lemma.

Lemma 8. Let $M \in \mathbb{R}^{n \times n}$ and $M_{s}:=1 / 2\left(M+M^{T}\right)$. Suppose $M$ is positive definite, and let $\beta>0$ be the smallest eigenvalue of $M_{s}$. Then for all $x \in \mathbb{R}^{n},\left\|M^{-1} x\right\| \leq \beta^{-1}\|x\|$.

Proof. $M^{-1}$ exists since $M_{s}$ is positive definite. Let $\nu_{\min }$ (resp. $\nu_{\max }$ ) be the smallest (resp. largest) singular values of $M$ (resp. $M^{-1}$ ). Two relations hold: $\nu_{\max }=\nu_{\min }^{-1}$ [29, Fact 6.3.28, p. 410] and $\nu_{\min } \geq \beta>0$ [30, Corollary 3.1.5, p.151]. Then using the spectral norm definition, $\left\|M^{-1} x\right\| \leq\left\|M^{-1}\right\|\|x\|=$ $\nu_{\max }\|x\| \leq \beta^{-1}\|x\|$.

From now on, let $C B_{s}^{*}:=1 / 2\left(C B^{*}+\left(C B^{*}\right)^{T}\right)$ and let $\beta$ be its smallest eigenvalue.

Proposition 2. Suppose that $C B^{*}$ is positive definite. If the controller gain $\alpha>0$ is such that for all $k \in \mathbb{N}$ : $\left\|C p_{k}\right\|<\alpha \beta$, then the perturbed closed-loop system (9) and (20) enters the discrete-time sliding phase in finite time and stays in it with $\bar{\sigma}_{k+1}=C p_{k}$. Furthermore if $h \in\left(0, h^{*}\right]$, as defined in Lemma 6 , then there exists an upper bound $T^{*}$ on the duration of the reaching phase.
Proof. Let $V\left(\bar{\sigma}_{k}\right):=\alpha\left\|\bar{\sigma}_{k}\right\|_{1}=-\bar{u}_{k-1}^{s T} \bar{\sigma}_{k},-\bar{u}_{k-1}^{s} \in \alpha \operatorname{Sgn}\left(\bar{\sigma}_{k}\right)$. Assume that the system is initialized outside the discretetime sliding phase. It follows that $\left\|\bar{u}_{k}^{s}\right\| \geq \alpha$. Starting from (18), doing as in the proof of Proposition 1 and adding the contribution of the perturbation, we have $V\left(\bar{\sigma}_{k+1}\right)-V\left(\bar{\sigma}_{k}\right) \leq$ $-\beta\left\|\bar{u}_{k}^{s}\right\|^{2}-\left(\bar{u}_{k}^{s}\right)^{T} C p_{k}$. Using the Cauchy-Schwarz inequality, we obtain $\left|\left(\bar{u}_{k}^{s}\right)^{T} C p_{k}\right| \leq\left\|\bar{u}_{k}^{s}\right\|\left\|C p_{k}\right\|$. To ensure that $V$ strictly decreases, we need $\left\|C p_{k}\right\|<\beta\left\|\bar{u}_{k}^{s}\right\|$. This condition is satisfied using the hypothesis on the gain $\alpha$ and the fact that $\beta>0$. Note that even in the case with multiple switching surfaces, $V$ decreases as long as the system is not "sliding" on the intersection of all the manifolds: $\left\|\bar{u}_{k}^{s}\right\|<\alpha$ can only hold when $\widetilde{\sigma}_{k+1}=0$. If $\widetilde{\sigma}_{k+1}=0$, then we enter the discrete-time sliding phase. The finite-time property is derived as in the proof of Proposition 1. Let $\kappa=\alpha \beta-\left\|C p_{k}\right\|$. By assumption, $\kappa>0$ holds, therefore in the reaching phase, $V$ decreases by at least $\kappa \alpha$ at each sampling period. Hence, $V\left(\bar{\sigma}_{k}\right)$ converges to 0 in finite-time. Now if the system is in the discrete-time sliding phase at $t_{k}$, then $\widetilde{\sigma}_{k+1}=0$ and $\bar{\sigma}_{k+1}=C p_{k}$. At time $t_{k+1}$, we have $\widetilde{\sigma}_{k+2}=C p_{k}+C B^{*} \bar{u}_{k+1}^{s}$. Let us show that $\bar{u}_{k+1}^{s}=-\left(C B^{*}\right)^{-1} C p_{k}$ is the unique solution to the generalized equation (9). With this value, $\widetilde{\sigma}_{k+2}=0$. Using Lemma 8 and the hypothesis, $\left\|\bar{u}_{k+1}^{s}\right\| \leq \beta^{-1}\left\|C p_{k}\right\|<\alpha$. Relations between norms yield $\left\|\bar{u}_{k+1}^{s}\right\|_{\infty}<\alpha$. Then $\bar{u}_{k+1}^{s} \in$ $(\alpha, \alpha)^{p} \subset \alpha \operatorname{Sgn}(0)$ and $\bar{u}_{k+1}^{s}$ is a solution to (9). With the hypothesis of the proposition, $C B^{*}$ is also a $\mathbf{P}$-matrix. Then Lemma 4 can be applied and yields the uniqueness property. Thus $\bar{u}_{k+1}^{s}=-\left(C B^{*}\right)^{-1} C p_{k}$ is the unique solution to (9) at time $t_{k+1}$ and by induction the system stays in the discrete-time sliding phase.

In the following, we suppose that $h \in\left(0, h^{*}\right]$. Let $\delta_{\text {max }}^{*}=\|\Delta\|=\left\|C B_{s}^{*} / h^{*}-C B_{s}\right\|$ (from the proof of Lemma 6) with $h=h^{*}$. From the expression of $k_{0}$ in the proof of Proposition 1, the duration of the reaching phase is $h k_{0}<\frac{V\left(\bar{\sigma}_{0}\right) h}{\beta \alpha^{2}}+h$. Note that $\beta / h$ is an eigenvalue of $C B_{s}^{*} / h$. Applying again Corollary 4.2.16, p. 405 in [26], we have $\lambda-\beta / h \leq \delta_{\max } \leq \delta_{\max }^{*}$, with $\lambda$ an eigenvalue of $C B_{s}$. This yields $\gamma-\delta_{\max }^{*} \leq \lambda-\delta_{\max }^{*} \leq \beta / h$. Using this in the previous inequality, we get $h k_{0}<\frac{V\left(\bar{\sigma}_{0}\right)}{\alpha^{2}\left(\gamma-\delta_{\max }^{*}\right)}+h^{*}=: T^{*}$.

Remark 3. In continuous time, the usual condition is $\alpha>$ $\|\xi\|_{\infty, \mathbb{R}_{+}}$. If the perturbation $\xi$ is continuous, then it is possible to link this condition to the one used in the previous theorem, $\left\|C p_{k}\right\|<\alpha \beta$ for all $k \in \mathbb{N}$. Using the mean value theorem for integration, we get $C p_{k}=h C e^{A\left(t_{k+1}-t^{\prime}\right)} B \xi\left(t^{\prime}\right)=$ $h C B \xi\left(t^{\prime}\right)+\mathcal{O}\left(h^{2}\right)$, with $t^{\prime} \in\left[t_{k}, t_{k+1}\right]$. Hence the first-order estimation for $\left\|C p_{k}\right\|$ is $h\|C B\|\|\xi\| \leq h \sqrt{p}\|C B\|\|\xi\|_{\infty, \mathbb{R}_{+}}$. From the proof of Lemma 6 , it follows that $\beta=h \lambda_{\min }(C B)+$ $\mathcal{O}\left(h^{2}\right)$, and from [30, Corollary 3.1.5, p.151] we have $\|C B\|>$ $\lambda_{\max }(C B)$. For $h$ small enough, we infer $\|C B\| \sqrt{p} / \beta \geq 1$ and therefore $\left\|C p_{k}\right\|<\alpha \beta$ implies $\alpha>\|\xi\|_{\infty, \mathbb{R}_{+}}$. If the sliding variable is a scalar, then the converse is also true at the limit.

Remark 4 . We studied the auxiliary system on the sliding variable $\bar{\sigma}$. We just provide a hint at the behavior of the original system (7). Let us use the following change of basis $P=\left(\begin{array}{c}C \\ N \Pi\end{array}\right)$, with $\Pi$ used in (5) with $\Pi B=0$ and $N \in \mathbb{R}^{(n-p) \times n}$ coming from the QR decomposition of $C=(Q N)\left(\begin{array}{l}R \\ 0\end{array}\right)$. Then from (7) the dynamics of $\eta:=N \Pi x$ are given by $\bar{\eta}_{k+1}=\bar{A}_{12} \bar{\sigma}_{k}+$ 
$\bar{A}_{22} \bar{\eta}_{k}$, with $\bar{A}=P e^{A h} P^{-1}$ and $\bar{A}_{12}$ and $\bar{A}_{22}$ arising from the block decomposition. Let us assume that the matrix $C$ is such that the dynamics with $\sigma_{k}=0$, given by $\bar{\eta}_{k+1}=\bar{A}_{22} \bar{\eta}_{k}$, is asymptotically stable. Then if the system is in the discretetime sliding phase, $\sigma_{k}$ remains bounded and may be seen as a perturbation in the dynamics of $\eta_{k}$. If the latter is stable, $\bar{\eta}_{k}$ ends up in a bounded ball, which radius depends on the magnitude of the perturbation and the norm of $\bar{A}_{12}$.

In the classical literature on discrete-time sliding mode, where the explicit discretization (23a) is used [1], [3], [7], two conditions related to the sliding variable emerged: $\left(\bar{\sigma}_{k+1}-\right.$ $\left.\bar{\sigma}_{k}\right)_{i}\left(\sigma_{k}\right)_{i}<0$ for all $i=1, \ldots, n$, which is necessary; and the second one is $\left|\left(\bar{\sigma}_{k+1}\right)_{i}\right|<\left|\left(\bar{\sigma}_{k}\right)_{i}\right|$. With our approach the conditions for linear systems, stated in Lemma 7, and Proposition 2 are on the system parameters and not on the evolution on the sliding variable, which derives from the dynamics. This is much closer to the stability results obtained in continuous-time [27].

Looking at the value of the sliding variable in the discretetime sliding phase, $\bar{\sigma}_{k}=C p_{k-1}$ implies by the definition of the right-hand side that $\left\|\bar{\sigma}_{k}\right\|_{\infty}$ has an upper bound proportional to $h\|\xi\|_{\infty}$. Let us now study the influence of the gain on the control input.

Corollary 1. Suppose that $\alpha$ is such that for all $k \in \mathbb{N}$, $\left\|C p_{k}\right\| \leq \alpha \beta$. Then even if the controller gain is increased to $\alpha^{\prime}>\alpha$, the control input $\bar{u}^{s}$ does not change in the discretetime sliding phase.

Proof. From the proof of Proposition 2, we have that $\bar{u}_{k}^{s}$ is uniquely defined as the solution to (9), and is equal to $-\left(C B^{*}\right)^{-1} C p_{k}$ which does not depend on the controller gain.

This is a major difference with the explicitly discretized controller, where a change in the controller gain always influences the control input. This result is also similar to the continuous-time case: within Filippov's framework, when the system is in the sliding phase, the control input is a selection of the set-valued right-hand side which does not depend on the gain, given that the latter is large enough to dominate the perturbation.

Remark 5. Let us highlight two similarities between the continuous-time and the discrete-time sliding mode controllers we present here: the first is the expression of the control input value during the sliding phase. In continuous-time, with Filippov's notion of solution, we have $u^{s}(t)=-\xi(t)$. In other words, the control input is the selection of the setvalued right-hand side which exactly compensates for the disturbance. With the implicit (discrete-time) controller, we have $\bar{u}_{k+1}^{s}=-\left(C B^{*}\right)^{-1} C p_{k}$ and $p_{k}$ is an integral term involving $\xi$, see (19). The connection between the two inputs is the topic of the next section. The other point is the existence of an upper bound on the reaching phase, which is denoted by $T^{*}$, as in continuous-time.

\section{Convergence of the control input}

Let us now turn our attention to the relationship between $u$ and $\bar{u}$. In particular, we study the convergence of $\bar{u}$ to $u$ during the discrete-time sliding phase, which is established after $T^{*}<+\infty$. To the best of our knowledge, the only convergence study of this type is in [31], with a slightly different approach but which requires the symmetry of $C B$. From now on, we consider that the perturbation $\xi$ is only time-dependent.

Proposition 3. Consider the discrete-time closed-loop system given by (20) and (9). Let $\left\{h_{n}\right\}_{n \in \mathbb{N}}$ be any strictly decreasing sequence of positive numbers converging to 0 and with $h_{0}<h^{*}$ (see Lemma 6). Suppose that the perturbation $\xi: \mathbb{R} \rightarrow \mathbb{R}^{p}$ is uniformly continuous, that $C B$ is positive definite and that $\alpha>0$ is chosen such that the conditions of Proposition 2 are satisfied for each sampling period $h_{n}$. Then for any interval $S \subseteq\left[T^{*}, \infty\right), \lim _{h_{n} \rightarrow 0}\left\|\bar{u}^{s}-u^{s}\right\|_{\infty, S}=0$.

The proof is in Appendix A. It is also interesting to study the convergence of the variation of the control variable, which may be thought of as a measure of the control input chattering. Let us first define the variation of a function in some special cases. The material in Definition 4 is adapted from [32].

Definition 4. Let $f: \mathbb{R} \rightarrow \mathbb{R}^{m}$ be a right-continuous step function, discontinuous at finitely many time instants $t_{k}$ and $t_{0}, T \in \mathbb{R}$ with $t_{0}<T$. The variation of $f$ on $\left[t_{0}, T\right]$ is defined as:

$$
\operatorname{Var}_{t_{0}}^{T}(f):=\sum_{k}\left\|f\left(t_{k}\right)-f\left(t_{k-1}\right)\right\|,
$$

with $k \in \mathbb{N}^{*}$ such that $t_{k} \in\left(t_{0}, T\right]$. If $f$ is continuously differentiable with bounded derivatives then the variation of $f$ on $\left[t_{0}, T\right]$ is defined as:

$$
\operatorname{Var}_{t_{0}}^{T}(f):=\int_{t_{0}}^{T}\|\dot{f}(\tau)\| \mathrm{d} \tau
$$

Proposition 4. Suppose that $C B$ is positive definite, and $\xi$ is a real-valued continuously differentiable with bounded derivative function. Let $\left\{h_{n}\right\}_{n \in \mathbb{N}}$ be any strictly decreasing sequence of positive numbers converging to 0 with $h_{0}<h^{*}$. Let $\alpha$ be chosen such that the conditions of Proposition 2 are satisfied for each $h_{n}$. Let $T>T^{*}$ with $T^{*}$ defined in Proposition 2. Then $\lim _{h_{n} \rightarrow 0} \operatorname{Var}_{T^{*}}^{T}\left(\bar{u}^{s}\right)=\operatorname{Var}_{T^{*}}^{T}\left(u^{s}\right)$.

The proof is in Appendix B. In order to compare with the control input given by the explicit discretization as in (23a) below, let us recall the conclusion from [10] and [33], valid for a 2-D linear system with an explicit discretization of the sgn function: if the sampling period is small enough, once the closed-loop system is close to the sliding manifold, it spends at most two consecutive sampling periods on each side of the sliding surface. The control input variation is easy to compute as the sgn function is equal to +1 or -1 : each time the sliding manifold is crossed, the variation increases by 2 . It is then easy to see that for a small enough sampling period, the variation of the explicitly discretized control grows linearly with the inverse of the sampling period, hence it explodes as $h \rightarrow 0$.

In Sections VI and VII, we illustrate some results presented in this section: Proposition 2 is verified in the next section and in Section VII alongside Proposition 3. 


\section{EXPERIMENTAL RESULTS}

Let us present some experimental results obtained with the implicit controller (15) of Section IV-C, with a focus on the sliding variable and the control input. They illustrate the stability and robustness results of the previous section. To put those results into perspective, we also provide data captured when the signum function is explicitly discretized. The main point is that the implicitly discretized controller yields much better performances in the sense that the mean of the absolute value of the sliding variable is much smaller than with the explicitly discretized one. The chattering on both the control input and the sliding variable is also drastically reduced. The explicit discrete-time controller has the following control input value: $u(t)=-(C B)^{-1} C A x\left(t_{k}\right)-\alpha \operatorname{sgn}\left(C x\left(t_{k}\right)\right)$ for $t \in\left[t_{k}, t_{k+1}\right)$, arising from the explicit discretization of the continuous-time one in (3) and (4). Further investigations on various discrete-time controllers, including this one, can be found in the next section. The system, on which the experiments were conducted, is an inverted pendulum on a cart, located in the CRIStAL laboratory, École Centrale de Lille, France. The actuator is a linear motor to which the pendulum is fixed. The mechanism is sketched on Fig. 1. We use the following linearized model around the unstable equilibrium $\mathbf{x}_{\mathrm{eq}}=\left(\begin{array}{llll}0 & 0 & 0 & 0\end{array}\right)^{T}$ :

$$
\begin{aligned}
& \left\{\begin{array}{l}
\dot{\mathbf{x}}=A \mathbf{x}+B u \\
y=H \mathbf{x}
\end{array} \quad \text { with } \mathbf{x}=\left(\begin{array}{c}
x \\
\dot{x} \\
\theta \\
\dot{\theta}
\end{array}\right),\right. \\
& A=\left(\begin{array}{cccc}
0 & 1 & 0 & 0 \\
0 & 0 & -\frac{m_{a}}{M} g & 0 \\
0 & 0 & 0 & 1 \\
0 & 0 & \frac{\left(M+m_{a}\right)}{M l} g & 0
\end{array}\right), B=\left(\begin{array}{c}
0 \\
\frac{a}{M} \\
0 \\
-\frac{a}{M l}
\end{array}\right), H^{T}=\left(\begin{array}{l}
1 \\
0 \\
1 \\
0
\end{array}\right) .
\end{aligned}
$$

with $M=3.9249 \mathrm{~kg}$ and $m_{a}=0.2047 \mathrm{~kg}$ the mass of the cart and the pendulum, $l=0.2302 \mathrm{~m}$ is the length of the pendulum, $g=9.81 \mathrm{~m} / \mathrm{s}^{2}$ is the gravitational constant and $a=25.3 \mathrm{~N} / \mathrm{V}$ is the motor gain. The control input $u$ is proportional to the input voltage of the linear motor. The position $x$ and the angle $\theta$ are available but both the speed $v$ and angular velocities $v_{\theta}$ are computed using a filtered differentiator with $z$-transfer functions $D(z)=\frac{z-1}{\tau(z-\exp (-h / \tau))}$. The use of the linearized model contributes to the uncertainties, which can be considered as matched. A complete model can be found in [34], which details also the two frictional torques on the cart and the pendulum. The first one is matched, but not the other one. However, the given numerical values are such that the first one is much larger than the second one, whose effect is considered negligible. This is validated by the small magnitude of the sliding variable during the experiments.

The control objective is to maintain the pendulum at the unstable equilibrium $\mathbf{x}_{\text {eq }}$. The sliding surface was designed using an LMI procedure presented in [35], and such that on the sliding manifold the non-zero eigenvalues of the closed-loop system are in a cone in the left-hand complex plane. This criteria is expected to reduce the oscillations on the sliding surface. The resulting sliding surface is $C=$ (1.38050 1.354714 .134100 .62497$)$. The experiments were done with an initial position close to the unstable equilibrium in order to avoid the additional complexity of a switching logic between a local sliding mode controller and global controller.
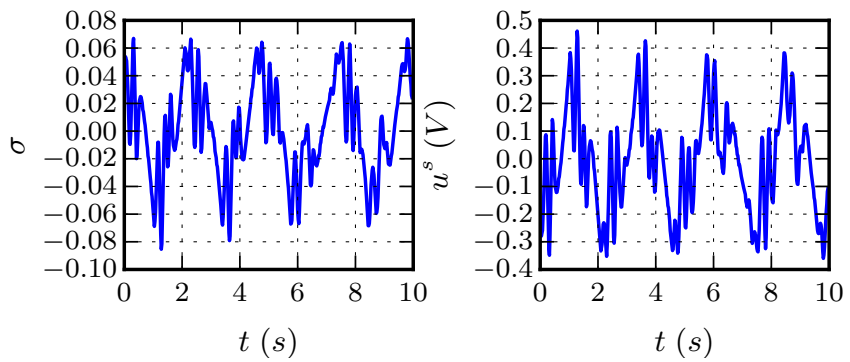

Fig. 2: Experiments: implicit controller with $h=20 \mathrm{~ms}, \alpha=1$.
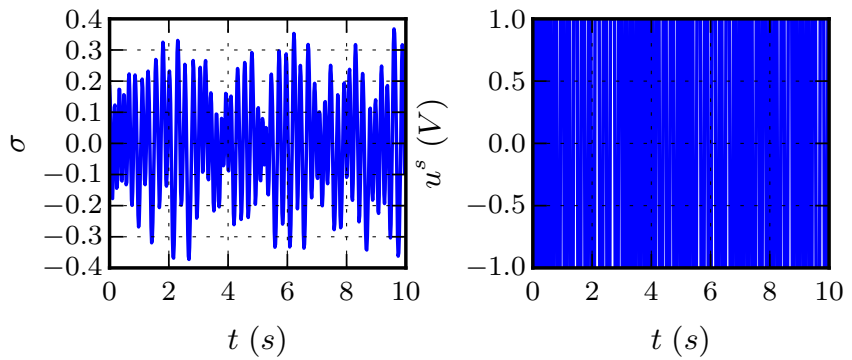

Fig. 3: Experiments: explicit controller with $h=20 \mathrm{~ms}, \alpha=1$.

Therefore the reaching phase is short or nonexistent and the closed-loop system is mostly in the discrete-time sliding phase with the controller (15).

Let us start with a comparison between two controllers which differ in the way their signum function was discretized: one was implicitly discretized (Fig. 2) and the other one explicitly (Fig. 3). In each case, the sliding variable and the discontinuous control input $u^{s}$ are depicted. With the sampling period set to $20 \mathrm{~ms}$, the scalar $C B^{*}$ is equal to 0.1978 ,



Fig. 1: Inverted pendulum on a cart. meaning that all the results from the previous section hold. Looking at the amplitudes of the sliding variable $\sigma$ in Fig. 2 and Fig. 3, it is clear that the implicitly discretized controller is able to maintain the value of the sliding variable an order of magnitude smaller than the explicitly discretized one. Looking at the control input, the difference is even more striking: on Fig. 2, the control input takes values that are proportional to the sliding variable and the control bounds are never reached, whereas in the explicit case Fig. 3, it is a high-frequency bang-bang input.

Let us now illustrate the meaning of the variation of a function introduced in Definition 4, by computing this quantity for both the control input and the sliding variable in Fig. 2 and Fig. 3. The figures are displayed in Table I: for the implicit controller in Fig 2, the variation is equal to 96.24 whereas in the explicit case (Fig 3) it is 1332.89. For the sliding variable, that is computing the output chattering, we get 3.10 in the implicit case and 44.74 in the explicit case. Hence in both cases the implicit discretization reduces the variation (or chattering) by one order of magnitude. The main cause of chattering in the explicit case is the bad discretization of the control input. In the implicit case, the numerical noise introduced by the 
filtered differentiators is the main source.

Table I: Control input and sliding variable variations with both the implicit and explicit controller.

\begin{tabular}{lll}
\hline Controller & $\operatorname{Var}_{0}^{10}(\bar{u})$ & $\operatorname{Var}_{0}^{10}(\bar{\sigma})$ \\
\hline Implicit & 96.24 & 3.10 \\
\hline Explicit & 1332.89 & 44.74 \\
\hline
\end{tabular}

Corollary 1 states that the implicit controller, in the discrete-
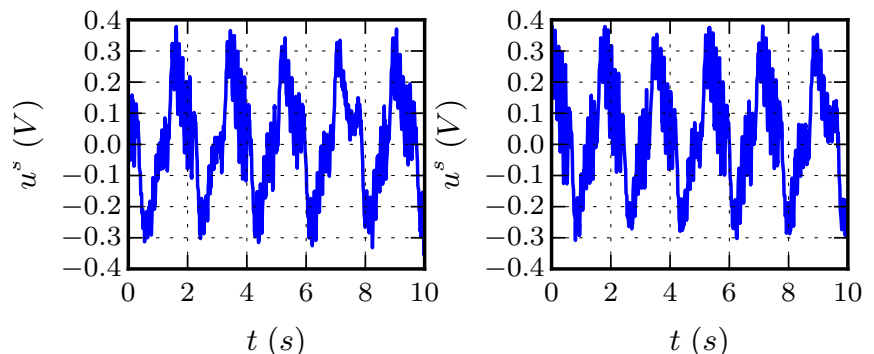

Fig. 4: Experiments: control input values with 2 different gains: $\alpha=1$ on the left and $\alpha=3$ on the right; $h=7 \mathrm{~ms}$.

time sliding phase, does not change if the gain is increased. On Fig. 4, this property is verified: the gain is increased threefold, but the bounds and shape of the control input are the same.

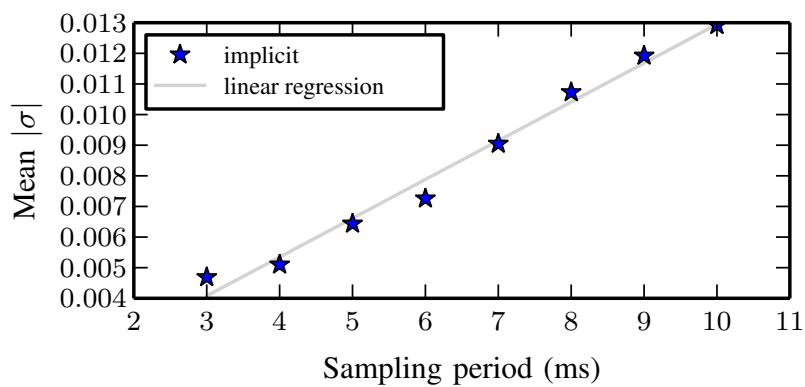

Fig. 5: Experiments: evolution of the precision with respect to the sampling period

To end this section, we quickly present another property of the closed-loop system with an implicit controller. Fig. 5 illustrates the good behavior of the controller when the sampling period is increased. We choose to define the precision of the controller as the mean of the absolute value of the measured values of $\sigma$. The linear regression, with slope 1.27 and $\mathrm{y}-$ intercept $2.77 \cdot 10^{-4}$ (close to $h^{2}=4 \cdot 10^{-4}$ ), indicates an evolution of the precision that is linear with respect to $h$. Given the value of the y-intercept, it looks like the precision is $\mathcal{O}(h)$. We investigate this property further in Section VIII-B2, where it is shown that this chattering is solely due to the perturbation.

The experimental data show that the implicitly discretized controller supersedes the explicitly discretized one. Both the input and output chattering are greatly reduced. This is further investigated with the numerical simulations in the next section.

\section{NUMERICAL ANALYSIS OF THE CONTROL INPUTS DISCRETIZATION}

In this section we present a numerical analysis of the influence on the closed-loop behavior with different types of discretization of both $u^{e q}$ and $u^{s}$ and we also illustrate some results from the previous section. In Section VIII some observations we make in this section are formalized, by providing theoretical results on the order of convergence for the different discretization methods. We consider a 2D system, in order to plot the state evolution, and which enables us to show a variety of behaviors. The next system is chosen for the simulations:

$$
\begin{cases}\dot{x}(t)=A x(t)+B \bar{u}(t) & A=\left(\begin{array}{cc}
0 & 1 \\
19 & -2
\end{array}\right), \\
\sigma=C x & B=\left(\begin{array}{l}
0 \\
1
\end{array}\right), C^{T}=\left(\begin{array}{l}
1 \\
1
\end{array}\right) . \\
\bar{u}(t)=\bar{u}^{e q}(t)+\bar{u}^{s}(t) & \end{cases}
$$

The matrix $\mathrm{A}$ has the eigenvalues $\lambda_{1}=3.47$ and $\lambda_{2}=-5.47$. The dynamics on the sliding surface is given by $\Pi A=\left(\begin{array}{cc}0 & 1 \\ 0 & -1\end{array}\right)$ (see Section III), which has eigenvalues 0 and -1 . Throughout this section, we chose $\alpha=1$. The initial state is $(-15,20)^{T}$. The first set of simulations uses a sampling period $0.3 \mathrm{~s}$ for the control and the second one a sampling period $0.03 \mathrm{~s}$. The simulations run for 150 s and were carried out with the open source SICONOS software package [36 $]^{1}$. Figures were created using Matplotlib [37].

Let us present simulation results not only for the controller studied in Section IV but also for some other inputs. The objective in this section is to provide an overview of the different behaviors of the closed-loop system when various discretization methods are used. A more formal study of their properties and performances is done in Section VIII. From all the possible time-discretization schemes, we focus on the onestep explicit, implicit, and midpoint ones. With the expressions for $u^{e q}$ and $u^{s}$ in (3) and (4), the proposed discretized values for the equivalent control $\bar{u}_{k}^{e q}$ are:

$$
\begin{aligned}
\bar{u}_{k, e}^{e q} & =-(C B)^{-1} C A \bar{x}_{k} & & \text { explicit input, } \\
\bar{u}_{k, i}^{e q} & =-(C B)^{-1} C A \bar{x}_{k+1} & & \text { implicit input, } \\
\bar{u}_{k, m}^{e q} & =1 / 2\left(\bar{u}_{k, e}^{e q}+\bar{u}_{k, i}^{e q}\right) & & \text { midpoint input }
\end{aligned}
$$

and the two possibilities for the discontinuous control $\bar{u}_{k}^{s}$ are:

$$
\begin{array}{ll}
\bar{u}_{k}^{s}=-\alpha \operatorname{sgn}\left(\bar{\sigma}_{k}\right) & \text { explicit input, } \\
\bar{u}_{k}^{s} \in-\alpha \operatorname{Sgn}\left(\bar{\sigma}_{k+1}\right) & \text { implicit input. }
\end{array}
$$

We use the single-valued signum function in (23a) since the case $\bar{\sigma}_{k}=0$ is not worth considering for explicit inputs. Moreover with the set-valued Sgn function, if $\bar{\sigma}_{k}=0$, then we would have $\operatorname{Sgn}\left(\bar{\sigma}_{k}\right) \in[-\alpha, \alpha]^{p}$ and there is no proper selection procedure to get a value for $\bar{u}_{k}^{s}$, whereas the selection procedure in the implicit case is presented in Section IV, with the resolution of generalized equations. A closed-loop formula for (22b) is given in (26). The most commonly used control law is the combination of (22a) and (23a). This kind of discretization has been studied in [9], [10], [38], with a focus on the sequence formed by $\bar{\sigma}_{k}$ once the system state approaches the sliding manifold. The value of (22b) is detailed in Section VIII-A. The ZOH sampled-data version of the system (21) is used for the discrete-time dynamics. In Section VII-A, the nominal system (21) is simulated and a matching perturbation is added in Section VII-B. More simulation results are available, with a systematic comparison between the implicit and explicit discretization of $u^{s}$, in [39].

\footnotetext{
${ }^{1}$ http://siconos.gforge.inria.fr
} 


\section{A. Nominal case}

The trajectories for the different closed-loop systems are plotted

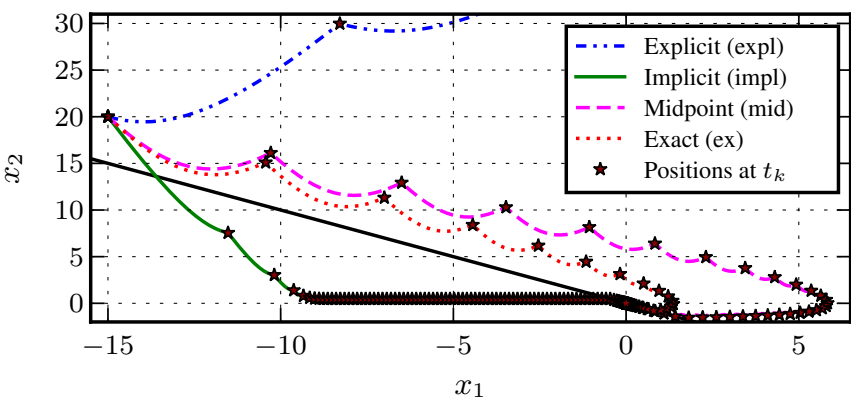

Fig. 6: Simulations of system (21) using different discretization methods for $u^{e q}$ and an implicit discretization of $u^{s}$, with $h=0.3 \mathrm{~s}$ and $\alpha=1$. Label (expl) is for pair (22a)-(23b); (impl) for (22b)-(23b); (mid) for (22c)-(23b); (ex) for (15).

in Fig. 6. The motion in the reaching phase depends only on the discretization method used for the equivalent control $u^{e q}$. It is only near the sliding manifold that the discretization method of the discontinuous control $u^{s}$ plays a role. Hence we only display the curves with an implicit discretization of $u^{s}$. If the explicit scheme in (22a) is used for the discretization of $u^{e q}$, the system diverges (curve (expl)): this discretization method can destabilize a system which is stable in continuous time. If the implicit scheme in (22b) is used for the discretization of $u^{e q}$, then the discretization error may not affect stability but it can induce some unexpected behavior. As we can see in Fig. 6, curve (impl), the trajectories are crossing the sliding manifold. This phenomenon can be explained by the following fact: let $\Delta_{k}$ be the discretization error on $u^{e q}$ at time $t_{k}$. We have the relation $\bar{\sigma}_{k+1}=\bar{\sigma}_{k}+\Delta_{k}+C B^{*} \bar{u}_{k}^{s}$. Let us consider the implicit discretization of $u^{s}$. If $0<\bar{\sigma}_{k}<C B^{*}$, then the system should enter the discrete-time sliding phase. However if $\Delta_{k}+\bar{\sigma}_{k}<-2 C B^{*}$, then for any value of $\bar{u}_{k}^{s}, \bar{\sigma}_{k+1}<-C B^{*}$. Hence, due to the discretization error, $\bar{u}^{s}$ fails to bring $\bar{\sigma}_{k+1}$ to 0 and the trajectory of the system crosses the sliding manifold. The same happens with the explicit discretization of $u^{s}$. With the midpoint method in (22c), curve (mid), and with the new control scheme (15), curve (ex), the system state reaches the sliding manifold directly.

Near the sliding manifold (Fig. 7a and 7b), the behavior of the system is more sensitive to the discretization of $u^{s}$. In the implicit case (method (23b), Fig. 7a) and in the discrete-time sliding phase, $\bar{\sigma}_{k}$ is very close to $0\left(\bar{\sigma}_{k}=0\right.$ with the exact method). In each case, the state converges to the origin (at the machine precision). This is visible on the zoom box in Fig. 7a, where markers indicate the state of the system at each time instant $t_{k}$, during the last second of each simulation. When the explicit method (23a) is used, the system chatters around the sliding manifold, within a neighborhood of order $h(0.3 \mathrm{~s}$ here), see Fig. $7 b$.

In Fig. 8b, the explicitly discretized discontinuous control $\bar{u}^{s}$ takes its values in $\{-1,1\}$ and starts at some point a limit cycle, as studied in [10]. This cycle is also visible on the zoom box in Fig. 7b with the help of the markers. In Fig. 8a, for each discretization of $u^{e q}, \bar{u}^{s}$ converges to 0 , which is the value that $u^{s}$ takes in the sliding phase. In the implicit and midpoint

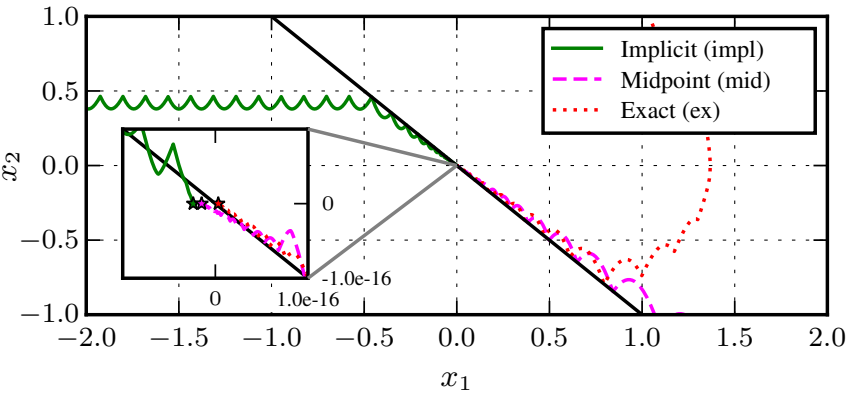

(a) Implicit discretization of $u^{s}$

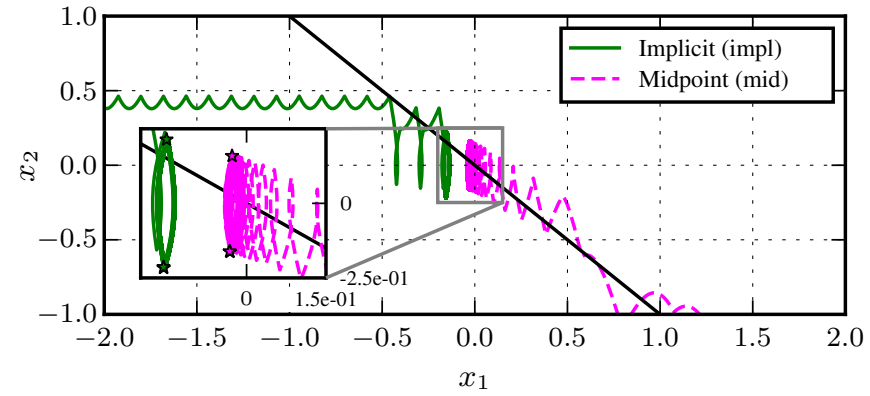

(b) Explicit discretization of $u^{s}$

Fig. 7: Detail of Fig. 6, with two discretizations of $u^{s}$.

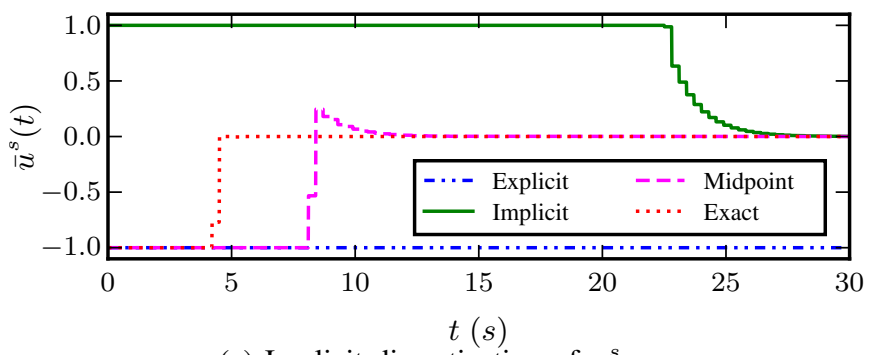

(a) Implicit discretization of $u^{s}$

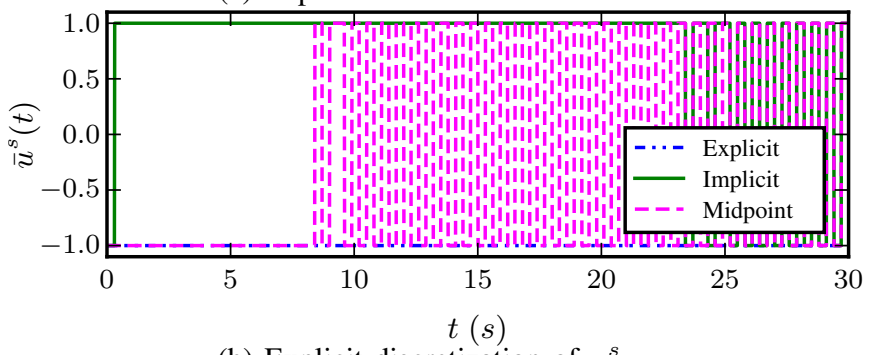

(b) Explicit discretization of $u^{s}$

Fig. 8: Evolution of $\bar{u}^{s}$ for different discretization methods, with $h=0.3 \mathrm{~s}$ and $\alpha=1$.

cases, at the beginning of the discrete-time sliding phase, $\bar{u}^{s}$ takes non zero values since there are discretization errors on $u^{e q}$. That is, if $\bar{\sigma}_{k}=0, \bar{\sigma}_{k+1} \neq 0$. The discontinuous control tries to bring $\bar{\sigma}_{k+1}$ to 0 and counteracts the effects of the error. As the state goes to the origin, the error converges to 0 . The simulation results seem to indicate that the discretization error is smaller in the midpoint case than in the implicit case. This observation is formally stated in Lemma 9 in the next section. With the exact method of Section IV-C, $\bar{u}^{s}$ goes to 0 after one sampling period in the discrete-time sliding phase. In Fig. 8a and $8 \mathrm{~b}$, with the explicit discretization of $u^{e q}, \bar{u}^{s}$ takes always the same value, since the closed-loop system moves away from the sliding manifold. In terms of convergence to the sliding 
manifold, the first closed-loop system to enter the discrete-time sliding phase is the exact method (Fig. 8a), then the midpoint, finally the implicit method. With the explicit method on $u^{e q}$, the system moves away from the sliding manifold and thus cannot enter the discrete-time sliding phase.

The results presented here bring into view the numerical chattering caused by an explicit discretization of $u^{s}$, while the implicit method is free of it. The importance of the discretization of $u^{e q}$ is also illustrated, with the explicit method leading to a diverging system and the counterintuitive behavior yielded by the implicit method. An analysis of the different phenomena is provided in the next section. The exact method from Section IV-C produces good results and in agreement with the theoretical results.

\section{B. Perturbed case}

We now add a perturbation in the system (21), which takes the form $\xi(t)=0.6 \exp (\min (6-t, 0)) \sin (2 \pi t)$ in the next set of simulations. Note that for all $t,|\xi(t)| \leq 0.6$. This particular $\xi$ has been chosen to highlight that if the perturbation vanishes, with the implicit discretization in (23b), $\bar{u}^{s}$ goes also to 0 , whereas in the explicit case (23a), $\bar{u}^{s}$ continues to switch between -1 and 1 . Recall that if the assumptions in Proposition 2 are satisfied, then $\bar{u}_{k}^{s}=-\left(C B^{*}\right)^{-1} C p_{k-1}$. It takes this value in order to counteract the effect of the perturbation during the elapsed time interval, hence imitating the solutions defined using Filippov's framework. However the trajectories are now clearly only in a neighborhood of the sliding manifold. Finally in each case in Fig. 9, $\bar{u}_{k}^{s}$ settles to 0 , as in continuous time. Indeed, the perturbation $\xi$ used in this simulation goes to 0 exponentially fast at some point. On the other hand, with an explicit discretization of $u^{s}$, the results are alike those displayed on Fig. 8b. It is much harder to see the influence of the perturbation on $\bar{u}^{s}$ since filtering would be necessary to see the effect. In Fig. 10 we further illustrate the

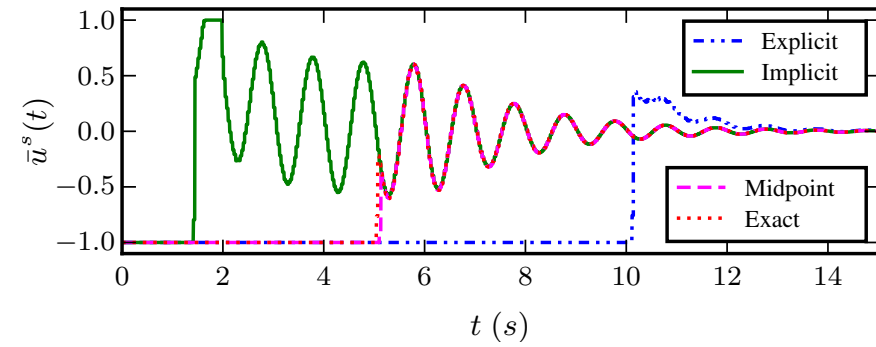

Fig. 9: Evolution of $\bar{u}^{s}$ for different discretization methods for $u^{e q}$ and an implicit discretization of $u^{s}, h=0.03 \mathrm{~s}$. (perturbed case)

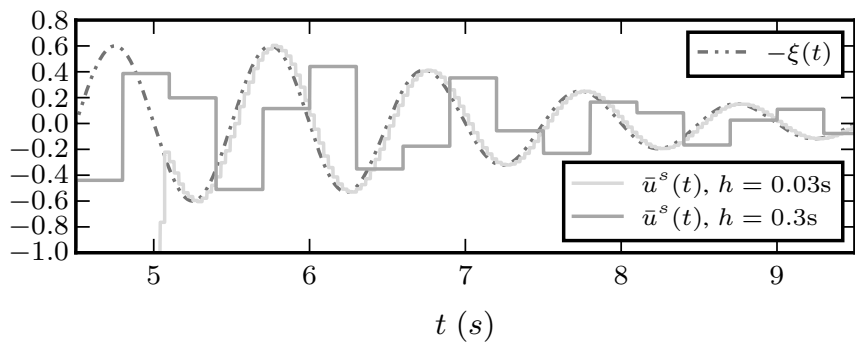

Fig. 10: Evolution of $\bar{u}^{s}$ and the perturbation using the new control scheme for two different sampling periods. phenomenon in the implicit case: $\bar{u}^{s}$ approximates $-\xi$ with a delay proportional to $h$. This illustrates the convergence of $\bar{u}^{s}$ to $u^{s}$, as stated in Proposition 3.

\section{DISCRETIZATION PERFORMANCE}

The aim of this section is to analyse the performances of the controllers presented in Section VII in (22a)-(22c) and (23a)(23b). The simulation results of the previous section indicate that the discretization method used to discretize the continuoustime controller has a clear incidence on the closed-loop behavior. We shall here provide results on the asymptotic behavior (as $h \rightarrow 0$ ) for both the equivalent and the discontinuous part of the control. In the last part, we discuss the case when there is a matched perturbation.

\section{A. Discretization of the state-continuous control}

Let us begin with the discretization error on $u^{e q}$ and more specifically on its effect on the sliding variable. In other words we analyze how the invariance property in (3) is preserved after discretization. In the following, $\bar{u}^{s}$ is set to 0 . Let $\Delta \bar{\sigma}_{k}:=$ $\bar{\sigma}_{k+1}-\bar{\sigma}_{k}$ be the local variation of the sliding variable due to the discretization error on $u^{e q}$.

Using an explicit discretization of $u^{e q}$ as in (22a), and using (7) the closed-loop discrete-time system dynamics is

$$
\bar{x}_{k+1}=\Phi_{k}^{e} \bar{x}_{k},
$$

with $\Phi_{k}^{e}:=e^{A h}-\Psi \Pi_{B} A, \Psi:=\int_{t_{k}}^{t_{k+1}} e^{A\left(t_{k+1}-\tau\right)} \mathrm{d} \tau=$ $\sum_{l=0}^{\infty} \frac{A^{l} h^{l+1}}{(l+1) !}$ and $\Pi_{B}:=B(C B)^{-1} C=I-\Pi$, where $\Pi$ is the projection operator from Section III. In the implicit case, the recurrence equation (7) combined with (22b) yields

$$
\bar{x}_{k+1}=e^{A h} \bar{x}_{k}-\Psi \Pi_{B} A \bar{x}_{k+1} \text { or } \bar{x}_{k+1}=W^{-1} e^{A h} \bar{x}_{k},
$$

with $W=I+\Psi \Pi_{B} A$. Starting from (22b), the control input value is

$$
\bar{u}_{k, i}^{e q}=-(C B)^{-1} C A W^{-1} e^{A h} \bar{x}_{k},
$$

which is non-anticipative.

Lemma 9. Let $\bar{u}^{s} \equiv 0$. With either an explicit or an implicit discretization of $u^{e q}$, the discretization error $\Delta \bar{\sigma}_{k}$ is $\mathcal{O}\left(h^{2}\right)$. If the midpoint method (22c) is used, then the error is $\mathcal{O}\left(h^{3}\right)$.

Proof. Let us start with the implicit case. There exists a Taylor expansion for $W^{-1}$ if $\Psi \Pi_{B} A$ has all its eigenvalues in the unit disk. Since $\Psi \rightarrow 0$ as $h \rightarrow 0$, it is always possible to find an $h_{0}$ such that this condition holds for all $h_{0}>h>0$. Since we are interested in an asymptotic property, such restriction on $h$ does not play any role. The finite expansion of $W^{-1} e^{A h}$ is:

$I-\left(\Pi_{B} A-A\right) h+\left(\frac{\Pi_{B} A \Pi_{B} A}{2}-\Pi_{B} A^{2}+\frac{A^{2}}{2}\right) h^{2}+\mathcal{O}\left(h^{3}\right)$.

The variation of the sliding variable is

$$
\begin{gathered}
\Delta \bar{\sigma}_{k}=\bar{\sigma}_{k+1}-\bar{\sigma}_{k}=C\left(W^{-1} e^{A h}-I\right) \bar{x}_{k} \\
=h(-A+A) \bar{x}_{k}+h^{2}\left(\frac{C A \Pi_{B} A}{2}-\frac{C A^{2}}{2}\right) \bar{x}_{k}+\mathcal{O}\left(h^{3}\right) \\
=\frac{h^{2}}{2} C A\left(I-\Pi_{B}\right) A \bar{x}_{k}+\mathcal{O}\left(h^{3}\right)=-\frac{h^{2}}{2} C A \Pi A \bar{x}_{k}+\mathcal{O}\left(h^{3}\right) .
\end{gathered}
$$


For the explicit case, expanding the exponential and $\Psi$ terms yields

$$
\Delta \bar{\sigma}_{k}=\frac{h^{2}}{2} C A \Pi A \bar{x}_{k}+\mathcal{O}\left(h^{3}\right) .
$$

With the midpoint method (22c), the recurrence equation is

$$
\bar{x}_{k+1}=0.5\left(e^{A h}-\Psi \Pi_{B} A\right) \bar{x}_{k}+0.5\left(e^{A h} \bar{x}_{k}-\Psi \Pi_{B} A \bar{x}_{k+1}\right) .
$$

The two terms are the right-hand side in (24) and (25). The term in $h^{2}$ is canceled and the error is of order $\mathcal{O}\left(h^{3}\right)$.

\section{B. Discretization of both control inputs}

In the following, we consider the sliding variable dynamics with the state-continuous and discontinuous set-valued controllers. It is expected that $\bar{\sigma}$ goes to 0 and once it reaches zero, stays at this value. The proposed metric to measure the performance of the discrete-time controller is the Euclidean norm of the sliding variable when the system state is close to the sliding manifold. Let $\varepsilon_{k}:=\left\|\bar{\sigma}_{k+1}\right\|$ be the discretization error when $\left\|\bar{\sigma}_{k}\right\|$ is small enough. We consider also the case with a matching perturbation (which is $\mathcal{O}(h)$ ), leading to dynamics as in (20).

1) Explicit discretization: In the sliding mode literature, several proposals (seven of them are listed in [40]) have been made to analyze the behavior of the closed-loop system near the sliding manifold and to propose new variable structure control strategies. Despite this, it is still difficult to analyze the behavior near the sliding manifold, besides stability. Thus we only study the invariance of a close neighborhood of the sliding manifold, also to provide an estimate of the chattering due to the discrete discontinuous control.

Lemma 10. Let the closed-loop system's state in (7) be in an $\mathcal{O}\left(h^{2}\right)$-neighborhood of the sliding manifold at $t=t_{k}$, i.e. $\bar{\sigma}_{k}=\mathcal{O}\left(h^{2}\right)$, but with $\bar{\sigma}_{k} \neq 0$. If the discontinuous part $u^{s}$ of the control is discretized using the explicit scheme (23a), then the discretization error $\varepsilon_{k}$ is $\mathcal{O}(h)$ and the system exits the $\mathcal{O}\left(h^{2}\right)$-neighborhood.

Proof. Starting from equation (7) and using the control inputs $\bar{u}_{k}^{e q}=-\left(C B^{*}\right)^{-1} C A \bar{x}_{k}$ and $\bar{u}_{k}^{s}=-\alpha \operatorname{sgn}\left(C \bar{x}_{k}\right)$, the following holds:

$$
\bar{\sigma}_{k+1}=C\left(e^{A h}-\Psi \Pi_{B} A\right) \bar{x}_{k}-C B^{*} \operatorname{sgn}\left(C \bar{x}_{k}\right)
$$

that is $\bar{\sigma}_{k+1}=\bar{\sigma}_{k}+\Delta_{k}-C B^{*} \operatorname{sgn}\left(\bar{\sigma}_{k}\right)$ with $\Delta_{k}:=C\left(e^{A h}-\right.$ $\left.I-\Psi \Pi_{B} A\right) \bar{x}_{k}$. Let us compute the square of the norm of the sliding variable, which is given by:

$$
\begin{aligned}
& \bar{\sigma}_{k+1}^{T} \bar{\sigma}_{k+1}=\left\|\bar{\sigma}_{k}\right\|^{2}+\left\|\Delta_{k}\right\|^{2}+\left\|C B^{*} \operatorname{sgn}\left(\bar{\sigma}_{k}\right)\right\|^{2} \\
& \quad+\bar{\sigma}_{k}^{T} \Delta_{k}-\bar{\sigma}_{k}^{T} C B^{*} \operatorname{sgn}\left(\bar{\sigma}_{k}\right)-\Delta_{k}^{T} C B^{*} \operatorname{sgn}\left(\bar{\sigma}_{k}\right) .
\end{aligned}
$$

From Lemma 9, we have $\left\|\Delta_{k}\right\|^{2}=\mathcal{O}\left(h^{4}\right)$. We can compute the order of the other terms with respect to $h$ :

$$
\begin{aligned}
\left\|C B^{*} \alpha \operatorname{sgn}\left(\bar{\sigma}_{k}\right)\right\|^{2} & =\left\|\sum_{k=0}^{\infty} h^{l+1} \frac{C A^{l} B}{(l+1) !} \alpha \operatorname{sgn}\left(\bar{\sigma}_{k}\right)\right\|^{2} \\
& \leq\left\|h C B \alpha \operatorname{sgn}\left(\bar{\sigma}_{k}\right)\right\|^{2}+\mathcal{O}\left(h^{3}\right) \\
\Delta_{k}^{T} C B^{*} \alpha \operatorname{sgn}\left(\bar{\sigma}_{k}\right) & =\mathcal{O}\left(h^{3}\right) .
\end{aligned}
$$

Using the Cauchy-Schwarz inequality on the remaining terms yields

$$
\begin{aligned}
\left|\bar{\sigma}_{k}^{T} \Delta_{k}\right| & \leq\left\|\bar{\sigma}_{k}\right\|\left\|\Delta_{k}\right\| \\
\left|\bar{\sigma}_{k}^{T} C B^{*} \operatorname{sgn}\left(\bar{\sigma}_{k}\right)\right| & \leq\left\|\bar{\sigma}_{k}\right\|\left\|C B^{*} \operatorname{sgn}\left(\bar{\sigma}_{k}\right)\right\| .
\end{aligned}
$$

If $\left\|\bar{\sigma}_{k}\right\|=\mathcal{O}\left(h^{2}\right)$, then the above terms are $\mathcal{O}\left(h^{4}\right)$ and $\mathcal{O}\left(h^{3}\right)$. Hence, the dominant term in (27) is $\left\|h C B \alpha \operatorname{sgn}\left(\bar{\sigma}_{k}\right)\right\|^{2}$. Let $\left\{\lambda_{i}\right\}$ be the spectrum of $h C B$, with $\lambda_{m}=\min _{i}\left|\lambda_{i}\right|$ and $\lambda_{M}=\max _{i}\left|\lambda_{i}\right|$. We have the following:

$$
\lambda_{m} h \alpha \sqrt{p} \leq\left\|h C B \operatorname{sgn}\left(\bar{\sigma}_{k}\right)\right\| \leq \lambda_{M} h \alpha \sqrt{p} .
$$

Inserting this in (27) yields that $\left\|\bar{\sigma}_{k+1}\right\|$ is $\mathcal{O}(h)$.

With a matched perturbation, the discrete-time dynamics of $\bar{\sigma}$ are given by (20). Then $\left\|\bar{\sigma}_{k+1}\right\|^{2}$ involves all the terms in (27), plus the following ones:

$$
\begin{aligned}
\left|\bar{\sigma}_{k}^{T} C p_{k}\right| \leq\left\|\bar{\sigma}_{k}\right\|\left\|C p_{k}\right\| & =\mathcal{O}\left(h^{3}\right) \text { by Cauchy-Schwarz } \\
\left|\Delta_{k}^{T} C p_{k}\right| \leq\left\|\Delta_{k}\right\|\left\|C p_{k}\right\| & =\mathcal{O}\left(h^{3}\right) \text { by Cauchy-Schwarz } \\
\left(C B^{*} \alpha \operatorname{sgn}\left(\bar{\sigma}_{k}\right)\right)^{T} C p_{k} & =\mathcal{O}\left(h^{2}\right) \\
\left\|C p_{k}\right\|^{2} & =\mathcal{O}\left(h^{2}\right) .
\end{aligned}
$$

Thus the dominant terms are $\left\|C p_{k}\right\|,\left\|C B^{*} \alpha \operatorname{sgn}\left(\bar{\sigma}_{k}\right)\right\|$ and $\left(C B^{*} \alpha \operatorname{sgn}\left(\bar{\sigma}_{k}\right)\right)^{T} C p_{k}$, all of them $\mathcal{O}(h)$. Those terms induce chattering and they all have the same order with respect to the sampling period $h$.

Therefore in the nominal case, with an explicit discretization of $u^{s}$, the main error comes from the discretization of the discontinuous control $u^{s}$, since it increases the error by an order $h$. When there is a matched perturbation, it also introduce terms in $\mathcal{O}(h)$. The dominant contribution is difficult to determine, a priori, but what remains is the (known) fact that the gain of the controller plays a role in the magnitude of the sliding variable.

2) Implicit discretization: In the following, we are interested in studying the discretization error in the same context as for the previous lemma.

Lemma 11. Let the closed-loop system be in the discrete-time sliding phase. In the nominal case, if the discontinuous part $u^{s}$ of the control is discretized using an implicit scheme, then the total discretization error $\varepsilon_{k}$ has the same order as the discretization error $\Delta \bar{\sigma}_{k}$ on $u^{e q}$ (that is $h^{2}$ for the methods (22a) and (22b), and $h^{3}$ for the midpoint method (22c)). If there is a matched perturbation, then the order is 1 and this increase of the order is due to the perturbation.

Proof. Let $\Delta_{k}=C\left(e^{A h}-I\right) x_{k}+C \Psi B \bar{u}_{k}^{e q}$, with $\bar{u}_{k}^{e q}$ given by any method in (22a)-(22c). The system is supposed to be in the discrete-time sliding phase, that is $u_{k}^{s} \in(-\alpha, \alpha)^{p}$. Then $\tilde{\sigma}_{k+1}=\bar{\sigma}_{k}+C B^{*} \bar{u}_{k}^{s}=0$. From (7) one has:

$$
\bar{\sigma}_{k+1}=\bar{\sigma}_{k}+\Delta_{k}+C B^{*} \bar{u}_{k}^{s}=\Delta_{k} .
$$

Let us go through all the possible discretizations of $u^{e q}$ : from Lemma 9, we know that in both the implicit and explicit cases, $\Delta_{k}$ is $\mathcal{O}\left(h^{2}\right)$ and with the midpoint method (22c) it is $\mathcal{O}\left(h^{3}\right)$. When there is a perturbation, we add $C p_{k}$ to (28):

$$
\bar{\sigma}_{k+1}=\bar{\sigma}_{k}+\Delta_{k}-C B^{*} \operatorname{sgn}\left(\bar{\sigma}_{k+1}\right)+C p_{k}=\Delta_{k}+C p_{k} .
$$


The chattering due to the perturbation is predominant and the sliding variable is $\mathcal{O}(h)$.

In Section V, conditions are derived to ensure that the system stays in the discrete-time sliding phase once it reaches it, with or without perturbation. With the controller from Section IV-C the discretization error $\Delta_{k}=0$ : in this case the chattering is solely due to the perturbation. This is what we observe on Fig. 5 from experimental results: the order 1 implies an asymptotic linear relation between $\bar{\sigma}$ and $h$, which is verified in the figure, even when the sampling is not too small.

\section{CONClusion}

In this article several properties of the implicitly discretized sliding mode controller are provided: Lyapunov stability of the discrete-time sliding variable, finite-time reachability of the sliding surface, convergence of the discrete-time control input to the continuous-time one and perturbation attenuation. Those results, mainly for the sliding variable, are valid for the closed-loop system thanks to a new discrete-time sliding mode control scheme. Both experimental results and numerical simulations (obtained with the open source INRIA software package SICONOS) illustrate those results and indicate that the use of an explicit discretization for the discontinuous part of the control yields numerical chattering. This is not the case when using an implicit discretization. We also consider several time discretizations of the classical ECB-SMC controller and underline the influence of the discretization method for the state-continuous part of the input: the use of an explicit method can make the closed-loop system diverge, whereas with the other methods it attains the sliding surface. Finally we provide a performance analysis of the different discretization methods, which highlights the ability of the implicit one to alleviate or suppress the numerical chattering. Note that the controller analyzed here has been implemented on a nonlinear setup. Two strategies have been used to get a linear relation as found in (9): feedback linearization [41], [42] and linearization around the current state [43]. Finally the relaxation of the knowledege of system parameters will be the object of future analysis.

\section{ACKNOWLEDGMENTS}

The authors would like to thanks the CRIStAL lab (UMR CNRS 9189) and especially Dr. Laurentiu Hetel for providing access to their inverted pendulum setup.

\section{APPENDIX A}

\section{PROOF OF PROPOSITION 3}

Proof. Let $\left\{t_{k}\right\}$ be a sequence such that for all $k \in \mathbb{N}$, $t_{k+1}-t_{k}=h_{n}$ with $t_{0}=\inf S$. For the sake of clarity, we omit to write explicitly the dependence on $n$. From Proposition 2 and Lemma 6, we know that for all $k$ such that $t_{k} \geq T^{*}, \bar{u}_{k}^{s}=$ $-\left(C B^{*}\right)^{-1} C p_{k-1}=-\left(C B^{*}\right)^{-1} C \int_{t_{k-1}}^{t_{k}} e^{A\left(t_{k}-\tau\right)} B \xi(\tau) \mathrm{d} \tau$. During the sliding phase, the continuous-time controller satisfies $u^{s}(t)=-\xi(t)$. Let $S$ be any time interval contained in $\left[T^{*},+\infty\right)$. Let $t \in S$ and $k \in \mathbb{N}$ is such that $t \in\left[t_{k}, t_{k+1}\right)$. Hence $\bar{u}^{s}(t)=\bar{u}_{k}^{s}$. Let us study $\bar{u}_{k}^{s}-u^{s}(t)$ :

$$
\bar{u}_{k}^{s}-u^{s}(t)=-\left(C B^{*}\right)^{-1} C \int_{t_{k-1}}^{t_{k}} e^{A\left(t_{k}-\tau\right)} B \xi(\tau) \mathrm{d} \tau+\xi(t)
$$

$$
=-\left(C B^{*}\right)^{-1}\left(\int_{t_{k-1}}^{t_{k}} C e^{A\left(t_{k}-\tau\right)} B \xi(\tau) \mathrm{d} \tau-C B^{*} \xi(t)\right) .
$$

Using (8), we obtain:

$$
\begin{aligned}
\bar{u}_{k}^{s}-u^{s}(t)=-\left(C B^{*}\right)^{-1} & \left(\int_{t_{k-1}}^{t_{k}} C e^{A\left(t_{k}-\tau\right)} B \xi(\tau) \mathrm{d} \tau\right. \\
& \left.-\int_{t_{k}}^{t_{k+1}} C e^{A\left(t_{k+1}-\tau\right)} B \xi(t) \mathrm{d} \tau\right) .
\end{aligned}
$$

With the change of variable $\tau^{\prime}=\tau+h_{n}$ in the first integral, we can group the two integrals in (29) as follows:

$$
\begin{aligned}
=- & \left(C B^{*}\right)^{-1}\left(\int_{t_{k}}^{t_{k+1}} C e^{A\left(t_{k+1}-\tau\right)} B\left(\xi\left(\tau-h_{n}\right)-\xi(t) \mathrm{d} \tau\right)\right. \\
= & -\left(C B^{*}\right)^{-1}\left(\int_{t_{k}}^{t_{k+1}} C B\left(\xi\left(\tau-h_{n}\right)-\xi(t)\right)\right. \\
& \left.+\sum_{l=1}^{\infty} \frac{C A^{l} B}{l !}\left(\left(t_{k+1}-\tau\right)^{l}\left(\xi\left(\tau-h_{n}\right)-\xi(t)\right)\right) \mathrm{d} \tau\right)^{(30)}
\end{aligned}
$$

Using again (8), the first factor can be approximated by:

$$
\begin{aligned}
\left(C B^{*}\right)^{-1} & =\left(h_{n} C B+\sum_{l=1}^{\infty} \frac{C A^{l} B}{(l+1) !} h_{n}^{l+1}\right)^{-1} \\
& =\left(I+\sum_{l=1}^{\infty} \frac{(C B)^{-1} C A^{l} B}{(l+1) !} h_{n}^{l}\right)^{-1}\left(h_{n} C B\right)^{-1} \\
= & \left(I-\frac{1}{2}(C B)^{-1} C A B h_{n}+\mathcal{O}\left(h_{n}^{2}\right)\right)\left(h_{n} C B\right)^{-1} .
\end{aligned}
$$

The Taylor expansion holds if $\sum_{l=1}^{\infty} \frac{(C B)^{-1} C A^{l} B}{(l+1) !} h_{n}^{l}$ has all its eigenvalues in the unit disk. This is a mere technical restriction, since it is always possible to find a small enough positive number $h_{n_{0}}$ such this condition is satisfied. Since we are interested in the case where $\left\{h_{n}\right\}$ converges to 0 , this requirement is supposed to hold. For the first term in the integrand in (30), we apply the mean value theorem for integration. If $\xi$ is a vector-valued function (this is the case when the sliding variable has dimension greater than 1 ), we apply the theorem for each component separately. This yields for $i=1, \ldots, n \quad\left(\int_{t_{k}}^{t_{k+1}} \xi\left(\tau-h_{n}\right)-\xi(t) \mathrm{d} \tau\right)_{i}=$ $h_{n}\left(\xi_{i}\left(t_{i}^{\prime}-h_{n}\right)-\xi_{i}(t)\right)$ for some $t_{i}^{\prime} \in\left[t_{k}, t_{k+1}\right]$. For the second part of the integrand in (30), we exchange the summation and integral signs. This is possible since the matrix exponential is normally convergent and $\xi$ is bounded on any interval $\left[t_{k}, t_{k+1}\right]$ ( $\xi$ is continuous). Moreover for all $l \geq 1$, with $\tau \in\left[t_{k}, t_{k+1}\right],\left(t_{k+1}-\tau\right)^{l}\left(\xi\left(\tau-h_{n}\right)-\xi(t)\right)=\mathcal{O}\left(h_{n}\right)$. Thus $\int_{t_{k}}^{t_{k+1}}\left(t_{k+1}-\tau\right)^{l}\left(\xi\left(\tau-h_{n}\right)-\xi(t)\right) \mathrm{d} \tau=\mathcal{O}\left(h_{n}^{2}\right)$. Then the difference $\bar{u}_{k}^{s}-u^{s}(t)$ in (30) can be rewritten as:

$$
-\left(I+\mathcal{O}\left(h_{n}\right)\right)\left(\int_{t_{k}}^{t_{k+1}} h_{n}^{-1}\left(\xi\left(\tau-h_{n}\right)-\xi(t)\right) \mathrm{d} \tau+\mathcal{O}\left(h_{n}\right)\right) .
$$

Taking the supremum norm and using standard estimation yields:

$$
\begin{aligned}
& \left.\left\|\bar{u}_{k}^{s}-u^{s}(t)\right\|_{\infty} \leq \| I+\mathcal{O}\left(h_{n}\right)\right) \|_{\infty} . \\
& \left(\max _{i} \sup _{t^{\prime} \in\left[t_{k}, t_{k+1}\right]}\left|\xi_{i}\left(t^{\prime}-h\right)-\xi_{i}(t)\right|+\left\|\mathcal{O}\left(h_{n}\right)\right\|_{\infty}\right)
\end{aligned}
$$




$$
\leq \max _{i} \sup _{t^{\prime} \in\left[t_{k}, t_{k+1}\right]}\left|\xi_{i}\left(t^{\prime}-h_{n}\right)-\xi_{i}(t)\right|+\mathcal{O}\left(h_{n}\right) .
$$

Since $\xi$ is uniformly continuous, for every $\varepsilon>0$, there exists $\delta>0$ such that for all $t_{1}, t_{2} \in \mathbb{R},\left|t_{1}-t_{2}\right| \leq \delta$ implies $\left\|\xi\left(t_{1}\right)-\xi\left(t_{2}\right)\right\| \leq \varepsilon$. Then the right-hand side of (32) converges to 0 as $h_{n} \rightarrow 0$. Since this is true for all $t \in S$, the proof is complete.

\section{APPENDIX B}

\section{PROOF OF PROPOSITION 4}

Proof. Let $\left\{t_{k}\right\}$ be a sequence such that for all $k \in \mathbb{N}, t_{k+1}-$ $t_{k}=h_{n}$ with $T^{*}+h_{n}>t_{0} \geq T^{*}$ and $N$ the largest integer such that $t_{N} \leq T$. Let us recall that, with the implicit controller defined by (20) and (9), the reaching phase duration is bounded and that the sliding phase is established at $t=T^{*}$ if $h_{n}$ is small enough. Recall that in continuous time, $u^{s} \equiv-\xi$ in the sliding phase. Let us study the difference between the variations of $\bar{u}^{s}$ and $u^{s}$, with $\Delta_{T}:=T-T^{*}$ :

$\left|\operatorname{Var}_{T^{*}}^{T}\left(\bar{u}^{s}\right)-\operatorname{Var}_{T^{*}}^{T}\left(u^{s}\right)\right|=\left|\sum_{k}\left\|\bar{u}_{k+1}^{s}-\bar{u}_{k}^{s}\right\|-\int_{T^{*}}^{T}\|\dot{\xi}(\tau)\| \mathrm{d} \tau\right|$.

With $w$ the modulus of continuity of $\|\dot{\xi}\|$, we get the estimation $[44, \mathrm{p} .52]$

$$
\leq \sum_{k}\left|\left\|\bar{u}_{k+1}^{s}-\bar{u}_{k}^{s}\right\|-h_{n}\left\|\dot{\xi}\left(t_{k}\right)\right\|\right|+\Delta_{T} w\left(h_{n}\right) .
$$

Using the reverse triangle inequality yields

$$
\leq \sum_{k}\left\|\bar{u}_{k+1}^{s}-\bar{u}_{k}^{s}-h_{n} \dot{\xi}\left(t_{k}\right)\right\|+\Delta_{T} w\left(h_{n}\right) .
$$

Expanding the difference $\bar{u}_{k+1}^{s}-\bar{u}_{k}^{s}$, we get

$$
\begin{aligned}
\bar{u}_{k+1}^{s} & -\bar{u}_{k}^{s}=\left(C B^{*}\right)^{-1}\left(\int_{t_{k}}^{t_{k+1}} C B\left(\xi(\tau)-\xi\left(\tau-h_{n}\right)\right) \mathrm{d} \tau\right. \\
& \left.+\int_{t_{k}}^{t_{k+1}} C\left(e^{A\left(t_{k+1}-\tau\right)}-I\right) B\left(\xi(\tau)-\xi\left(\tau-h_{n}\right)\right) \mathrm{d} \tau\right) .
\end{aligned}
$$

Using the approximation for $\left(C B^{*}\right)^{-1}$ in (31) yields

$$
\begin{gathered}
\bar{u}_{k+1}^{s}-\bar{u}_{k}^{s}-h_{n} \dot{\xi}\left(t_{k}\right)=\left(I+\mathcal{O}\left(h_{n}\right)\right) \cdot \\
\left(\int_{t_{k}}^{t_{k+1}} h_{n}^{-1}\left(\xi(\tau)-\xi\left(\tau-h_{n}\right)\right)-\dot{\xi}\left(t_{k}\right) \mathrm{d} \tau+\right. \\
\left.\int_{t_{k}}^{t_{k+1}}\left(h_{n} C B\right)^{-1} C\left(e^{A\left(t_{k+1}-\tau\right)}-I\right) B \xi(\tau)-\xi\left(\tau-h_{n}\right) \mathrm{d} \tau\right) .
\end{gathered}
$$

The first integral can be transformed into

$$
\int_{t_{k}}^{t_{k+1}} h_{n}^{-1}\left(\xi(\tau)-\xi\left(\tau-h_{n}\right)\right)-\dot{\xi}(\tau) \mathrm{d} \tau+\int_{t_{k}}^{t_{k+1}} \dot{\xi}(\tau)-\dot{\xi}\left(t_{k}\right) \mathrm{d} \tau
$$

With $w^{\prime}$ the modulus of continuity of $\dot{\xi}$, the second integral can be bounded by $h_{n} w\left(h_{n}\right)$. Using standard estimation inequalities, we obtain the following estimate for the second integral in (33):

$$
\begin{aligned}
& \left\|\int_{t_{k}}^{t_{k+1}} C\left(e^{A\left(t_{k+1}-\tau\right)}-I\right) B h_{n}^{-1}\left(\xi(\tau)-\xi\left(\tau-h_{n}\right)\right) \mathrm{d} \tau\right\| \\
& \quad \leq \int_{t_{k}}^{t_{k+1}}\|C\|\|B\|\left(e^{\|A\| h_{n}}-1\right) h_{n}^{-1}\left\|\xi(\tau)-\xi\left(\tau-h_{n}\right)\right\| \mathrm{d} \tau .
\end{aligned}
$$

Let us now come back to our main computation

$$
\begin{aligned}
& \left|\operatorname{Var}_{T^{*}}^{T}\left(\bar{u}^{s}\right)-\operatorname{Var}_{T^{*}}^{T}\left(u^{s}\right)\right| \leq \Delta_{T} w\left(h_{n}\right) \\
& +\left(1+\mathcal{O}\left(h_{n}\right)\right)\left(\int_{t_{0}}^{t_{N}} h_{n}^{-1}\left\|\xi(\tau)-\xi\left(\tau-h_{n}\right)-h_{n} \dot{\xi}(\tau)\right\| \mathrm{d} \tau\right. \\
& +\left(t_{N}-t_{0}\right) w^{\prime}\left(h_{n}\right)+\left\|(C B)^{-1}\right\|\|C\|\|B\|\left(e^{\|A\| h_{n}}-1\right) . \\
& \left.\int_{t_{0}}^{t_{N}} h_{n}^{-1}\left\|\xi(\tau)-\xi\left(\tau-h_{n}\right)\right\| \mathrm{d} \tau\right)
\end{aligned}
$$

The continuity of $\xi, \dot{\xi}$ and $\|\cdot\|$ allows us to use the mean value theorem for integration on both integrals:

$$
\begin{aligned}
& \int_{t_{0}}^{t_{N}} h_{n}^{-1}\left\|\xi(\tau)-\xi\left(\tau-h_{n}\right)-h_{n} \dot{\xi}(\tau)\right\| \mathrm{d} \tau= \\
& h_{n}^{-1}\left(t_{N}-t_{0}\right)\left\|\xi\left(\tau^{\prime}\right)-\xi\left(\tau^{\prime}-h_{n}\right)-h_{n} \dot{\xi}\left(\tau^{\prime}\right)\right\| .
\end{aligned}
$$

Using Taylor's theorem with the remainder in its Lagrange form on $\xi$, we can write $\xi\left(\tau^{\prime}-h_{n}\right)=\xi\left(\tau^{\prime}\right)-h_{n} \dot{\xi}\left(\tau^{\prime}\right)+h_{n}^{2} \dot{\xi}\left(\tau_{t}\right)$, with $\tau_{t} \in\left[\tau^{\prime}-h_{n}, \tau^{\prime}\right]$. Hence, this integral is $\mathcal{O}\left(h_{n}\right)$. Switching to the second one, we have

$$
\begin{aligned}
& \int_{t_{0}}^{t_{N}} h_{n}^{-1}\left\|\xi(\tau)-\xi\left(\tau-h_{n}\right)\right\| \mathrm{d} \tau= \\
& \quad\left(t_{N}-t_{0}\right) h_{n}^{-1}\left\|\xi\left(\tau^{\prime \prime}\right)-\xi\left(\tau^{\prime \prime}-h_{n}\right)\right\| \underset{h_{n} \rightarrow 0}{\longrightarrow}\left\|\dot{\xi}\left(\tau^{\prime \prime \prime}\right)\right\| \leq M
\end{aligned}
$$

for some $\tau^{\prime}, \tau^{\prime \prime}, \tau^{\prime \prime \prime} \in\left[t_{0}, t_{N}\right]$. Since $e^{\|A\| h_{n}}-1=\mathcal{O}\left(h_{n}\right)$, this part of (34) is $\mathcal{O}\left(h_{n}\right)$. Hence, we can rewrite (34) as

$\left|\operatorname{Var}_{T^{*}}^{T}\left(\bar{u}^{s}\right)-\operatorname{Var}_{T^{*}}^{T}\left(u^{s}\right)\right| \leq \Delta_{T} w\left(h_{n}\right)+\Delta_{T} w^{\prime}\left(h_{n}\right)+\mathcal{O}\left(h_{n}\right)$,

since $t_{N}-t_{0} \leq \Delta_{T}$. This completes the proof since $w$ and $w^{\prime}$ vanish as their argument tends to 0 .

\section{REFERENCES}

[1] S. Sarpturk, Y. Istefanopulos, and O. Kaynak, "On the stability of discrete-time sliding mode control systems," Automatic Control, IEEE Transactions on, vol. 32, no. 10, pp. 930-932, 1987.

[2] S. Drakunov and V. Utkin, "On discrete-time sliding modes," in Proc. of IFAC Nonlinear Control System Design Conf., 1989, pp. 273-278.

[3] K. Furuta, "Sliding mode control of a discrete system," Systems \& Control Letters, vol. 14, no. 2, pp. 145-152, 1990.

[4] V. Utkin, "Sliding mode control in discrete-time and difference systems," in Variable Structure and Lyapunov Control, ser. Lecture Notes in Control and Information Sciences. Springer, 1994, vol. 193, pp. 87-107.

[5] W. Gao, Y. Wang, and A. Homaifa, "Discrete-time variable structure control systems," Industrial Electronics, IEEE Transactions on, vol. 42 , no. 2, pp. 117-122, 1995.

[6] G. Golo and C̆. Milosavljević, "Robust discrete-time chattering free sliding mode control," Systems \& Control Letters, vol. 41, no. 1, pp. 19-28, 2000. 
[7] C̆. Milosavljević, "General conditions for the existence of a quasi-sliding mode on the switching hyperplane in discrete variable structure systems," Automation and Remote Control, vol. 46, no. 3, pp. 307-314, 1985.

[8] S. Qu, X. Xia, and J. Zhang, "Dynamics of discrete-time sliding-modecontrol uncertain systems with a disturbance compensator," Industrial Electronics, IEEE Transactions on, vol. 61, no. 7, pp. 3502-3510, 2014

[9] Z. Galias and X. Yu, "Complex discretization behaviors of a simple sliding-mode control system," Circuits and Systems II: Express Briefs, IEEE Transactions on, vol. 53, no. 8, pp. 652-656, 2006.

[10] _ "Analysis of zero-order holder discretization of two-dimensional sliding-mode control systems," Circuits and Systems II: Express Briefs, IEEE Transactions on, vol. 55, no. 12, pp. 1269-1273, 2008.

[11] B. Wang, X. Yu, and G. Chen, "ZOH discretization effect on single-input sliding mode control systems with matched uncertainties," Automatica, vol. 45, no. 1, pp. 118-125, 2009.

[12] V. Acary and B. Brogliato, "Implicit Euler numerical scheme and chattering-free implementation of sliding mode systems," Systems \& Control Letters, vol. 59, no. 5, pp. 284-293, 2010.

[13] V. Acary, B. Brogliato, and Y. Orlov, "Chattering-free digital slidingmode control with state observer and disturbance rejection," Automatic Control, IEEE Transactions on, vol. 57, no. 5, pp. 1087-1101, 2012.

[14] F. Plestan, V. Bregeault, A. Glumineau, Y. Shtessel, and E. Moulay, "Advances in high order and adaptive sliding mode control-theory and applications," in Sliding Modes after the First Decade of the 21st Century, ser. Lecture Notes in Control and Information Sciences. Springer, 2012, vol. 412, pp. 465-492.

[15] M. Defoort, T. Floquet, A. Kokosy, and W. Perruquetti, "A novel higher order sliding mode control scheme," Systems \& Control Letters, vol. 58, no. 2, pp. 102-108, 2009.

[16] W.-C. Su, S. Drakunov, and Ü. Özgüner, "Implementation of variable structure control for sampled-data systems," in Robust Control via Variable Structure and Lyapunov Techniques. Springer, 1996, pp. 87106.

[17] A. F. Filippov, Differential Equations with Discontinuous Righthand Sides, ser. Mathematics and Its Applications. Kluver Academic Publishers, 1988, vol. 18

[18] F. Callier and C. Desoer, Linear System Theory, ser. Springer Texts in Electrical Engineering. Springer New York, 1991.

[19] R. Cottle, J.-S. Pang, and R. Stone, The Linear Complementarity Problem, ser. Classics in Applied Mathematics. Society for Industrial Mathematics, 2009, no. 60.

[20] C. Edwards and S. Spurgeon, Sliding Mode Control: Theory and Applications, ser. Systems and Control Book Series. CRC Press, 1998, vol. 7.

[21] F. Facchinei and J.-S. Pang, Finite-Dimensional Variational Inequalities and Complementarity Problems, Volume I, ser. Springer Series in Operations Research. Springer, 2003.

[22] - Finite-Dimensional Variational Inequalities and Complementarity Problems, Volume II, ser. Springer Series in Operations Research. Springer, 2003.

[23] V. Acary and B. Brogliato, Numerical Methods for Nonsmooth Dynamical Systems: Applications in Mechanics and Electronics, ser. Lecture Notes in Applied and Computational Mechanics. Springer Berlin Heidelberg, 2008, vol. 35 .

[24] C.-F. Lin and W.-C. Su, "A total chattering-free sliding mode control for sampled-data systems," in American Control Conference, Proceedings of the 2004. IEEE, 2004, pp. 1940-1945.

[25] R. T. Rockafellar and R. J.-B. Wets, Variational Analysis, ser. Grundlehren der mathematischen Wissenschaften. Springer Berlin Heidelberg, 2009, vol. 317.

[26] D. Hinrichsen and A. J. Pritchard, Mathematical Systems Theory I, ser. Texts in Applied Mathematics. Springer Berlin Heidelberg, 2005, vol. 48.

[27] V. Utkin, Sliding Modes in Control and Optimization, ser. Communications and Control Engineering. Springer Berlin, 1992.

[28] P. Pepe, "Stabilisation in the sample-and-hold sense of nonlinear retarded systems," SIAM Journal on Control and Optimization, vol. 52, no. 5, pp 3053-3077, 2014.

[29] D. Bernstein, Matrix Mathematics. Princeton University Press, 2009.

[30] R. A. Horn and C. R. Johnson, Topics in Matrix Analysis. Cambridge University Press, 1991.

[31] J. Rulla, "Error analysis for implicit approximations to solutions to Cauchy problems," SIAM Journal on Numerical Analysis, vol. 33, no. 1 , pp. 68-87, 1996.

[32] L. Ambrosio, N. Fusco, and D. Pallara, Functions of Bounded Variation and Free Discontinuity Problems. Clarendon Press Oxford, 2000.
[33] S. Qu, X. Xia, and J. Zhang, "Dynamical behaviors of an Euler discretized sliding mode control systems," Automatic Control, IEEE Transactions on, vol. 59, no. 9, pp. 2525-2529, September 2014.

[34] S. Riachy, Y. V. Orlov, T. Floquet, R. Santiesteban, and J.-P. Richard, "Second-order sliding mode control of underactuated mechanical systems I: Local stabilization with application to an inverted pendulum," International Journal of Robust and Nonlinear Control, vol. 18, no. 4-5, pp. 529-543, 2008.

[35] L. Hetel, E. Fridman, and T. Floquet, "Variable structure control with generalized relays: A simple convex optimization approach," Automatic Control, IEEE Transactions on, vol. 60, no. 2, pp. 497-502, Feb 2015.

[36] V. Acary, B. Bremond, O. Huber, and F. Pérignon, "An introduction to SICONOS," INRIA, Rapport Technique RT-0340, 2015. [Online]. Available: http://hal.inria.fr/inria-00162911

[37] J. D. Hunter, "Matplotlib: a 2D graphics environment," Computing in Science \& Engineering, pp. 90-95, 2007.

[38] B. Wang, X. Yu, and X. Li, "ZOH discretization effect on higher-order sliding-mode control systems," Industrial Electronics, IEEE Transactions on, vol. 55, no. 11, pp. 4055-4064, 2008.

[39] O. Huber, V. Acary, and B. Brogliato, "Comparison between explicit and implicit discrete-time implementations of sliding-mode controllers," in Decision and Control (CDC), 2013 IEEE 52nd Annual Conference on, Dec 2013, pp. 2870-2875.

[40] K. Furuta and Y. Pan, "Discrete-time variable structure control," in Variable Structure Systems: Towards the 21st Century, ser. Lecture Notes in Control and Information Sciences, Y. X. and J.-X. Xu, Eds. Springer Berlin Heidelberg, 2002, vol. 472, pp. 57-81.

[41] B. Wang, B. Brogliato, V. Acary, A. Boubakir, and F. Plestan, "Experimental comparisons between implicit and explicit implementations of discrete-time sliding mode controllers: Toward input and output chattering suppression," IEEE Transactions on Control Systems Technology, vol. 23 , no. 5, pp. 2071-2075, 2015, doi: 10.1109/TCST.2015.2396473.

[42] O. Huber, B. Brogliato, V. Acary, A. Boubakir, F. Plestan, and B. Wang, "Experimental results on implicit and explicit time-discretization of equivalent-control-based sliding-mode control," in Recent Trends in Sliding-Mode Control, L. Fridman, J. Barbot, and F. Plestan, Eds. IET, The Institution of Engineering and Technology, 2016.

[43] O. Huber, V. Acary, B. Brogliato, and F. Plestan, "Discrete-time twisting controller without numerical chattering: analysis and experimental results with an implicit method," in Decision and Control (CDC), 2014 IEEE 53rd Annual Conference on, Dec 2014, pp. 4373 - 4378.

[44] P. J. Davis and P. Rabinowitz, Methods of Numerical Integration, 2nd ed. Academic Press, 1984. 